\title{
Conforming hierarchical basis functions
}

\author{
M J Bluck* \\ Department of Mechanical Engineering, Imperial College, London, SW7 2AZ. UK.
}

\begin{abstract}
A unified process for the construction of hierarchical conforming bases on a range of element types is proposed based on an ab initio preservation of the underlying cohomology. This process supports not only the most common simplicial element types, as are now well known, but is generalised to squares, hexahedra, prisms and importantly pyramids. Whilst these latter cases have received (to varying degrees) attention in the literature, their foundation is less well developed than for the simplicial case. The generalisation discussed in this paper is effected by recourse to basic ideas from algebraic topology (differential forms, homology, cohomology, etc) and as such extends the fundamental theoretical framework established by the work of Hiptmair [16-18] and Arnold et al. [4] for simplices. The process of forming hierarchical bases involves a recursive orthogonalisation and it is shown that the resulting finite element mass, quasi-stiffness and composite matrices exhibit exponential or better growth in condition number.
\end{abstract}

AMS subject classifications: 65M60, 65M38, 41A10

Key words: Finite elements, vector basis functions, differential forms.

\section{Introduction}

High order curl and div conforming finite and boundary elements have become increasingly popular over recent years due to the demand for accurate and efficient solvers in, for, example, electromagnetics. Generally, these higher order schemes provide higher convergence rates and are correspondingly more economical than their lower order counterparts. For problems with singular solutions, however, the convergence rate is independent of the order of these bases if elements with uniform size and polynomial order are employed. This makes the use of adaptive (both in terms of element size $(h)$ and order $(p)$ ) schemes highly attractive. This paper concerns the construction of hierarchical conforming basis functions, which are essential to the development of such adaptive strategies.

High order conforming bases on simplices, squares and hexahedra were proposed as far back as 1980 [24], comprising unisolvent spaces together with degrees of freedom identified with submanifolds. Numerous variations on this theme followed, eg $[10,14]$. In

${ }^{*}$ Corresponding author. Email address: m.bluck@imperial .ac.uk (M. Bluck) 
many ways these constructions were founded on specific realisations of Nédélec's original idea.

Following the early work of Bossavit [7,8], algebraic topology (AT) (principally differential forms, homology and cohomology) provided the essential tools on which such conforming bases could be placed on a sound theoretical footing. Subsequently these techniques were further developed by Hiptmair [16-18], Bossavit [9] and notably Arnold et al. [4] where the theoretical foundations for spaces on simplices are fully developed for conforming spaces of arbitrary dimension. The construction of spaces on other element geometries (squares, hexahedra, prisms, pyramids) has received less attention. Although constructions exist, these rarely follow the AT framework, relying instead on Nédélec's original prescription. Exceptions to this are Bluck et al. [5] in 2D. Importantly, the desire for geometric flexibility in finite element methods leads to the need for schemes which employ both tetrahedra and hexahedra. This necessarily leads to a requirement for prismatic and pyramidal elements. Development of conforming bases on pyramids is rare and most notable in this context is the work of Gradinaru \& Hipmair [12] and Graglia et al. [13]. Such functions are not wholly polynomial in nature and as a result are not directly amenable to construction via the Koszul operator employed by Arnold et al.

The development of hierarchical conforming bases is relatively recent, with much of the early work due to Webb [27], Andersen et al. [2,3] in the context of curl-conforming simplicial finite elements. Hiptmair [16] briefly discusses the construction of hierarchical bases in terms of spaces of differential forms, again applied to simplices. Ilic \& Notaros [19] develop curl-conforming hierarchical bases on curvilinear hexahedra, although poor conditioning is an issue. This issue of poor conditioning of the resulting matrix equations frequently arises in the development of hierarchical schemes. Indeed the bases developed by Webb are also very poorly conditioned. Techniques to address this have been the subject of much recent work, notably Ilic \& Notaros [20], Ingelstrom [21,22] for multilevel FE solvers using simplicial elements, Abdul-Rahman at al. [1] and Schöberl [25]. It has been shown by Graglia et al. [15] that hierarchical curl conforming bases can be classified as type A, B or C according to their approximation properties. Most recently Xin \& Cai [28] (for triangles) and Xin et al. [29] (for tetrahedra) have investigated the dependence of condition number on element order for certain type A hierarchical curl-conforming basis functions. In the work presented here, we will develop a very general construction on a wide range of element types and for both 1 and 2-forms (curl and div conforming respectively). We will follow the Graglia classification (where appropriate), extending them to 2-form cases and perform a conditioning analysis analogous to [29] for all element types.

The contributions contained in this paper are as follows:

1. The construction of conforming spaces of differential forms valid for all element types (simplices, hexahedra, prisms and pyramids) and of all orders, based on an $\mathrm{ab}$ initio preservation of the underlying cohomology.

2. Novel spaces for pyramids (essential for a conforming representation on mixed element geometries) which are piecewise polynomial in nature, allowing an applica- 
tion of the Koszul operator.

3. A general (ie non element-type specific) geometric decomposition of these function spaces over submanifolds, allowing continuity of trace between all element types.

4. An algorithm for the construction of corresponding hierarchical bases up to arbitrary order.

5. It is shown that these bases give rise to mass, quasi-stiffness and composite matrices which grow exponentially or better with polynomial degree. In particular, tetrahedra and hexahedra exhibit consistently better conditioning than both prisms and pyramids.

In section 2 we construct (directly from the cohomological requirements) canonical function spaces on a wide range of element geometries of all orders. For the case of simplices, this is merely a special case (in 2 and 3 dimensions) of the work of Arnold et al. and is included for completeness. For tensor product spaces (squares, hexahedra, prisms), whilst an approach based on the application of the Koszul operator is entirely feasible, we instead make use of the Künneth formula - again a basic tool in AT as this leads much more directly to the corresponding canonical spaces. For the pyramidal case, the Koszul operator is applied to a recently developed class of piecewise polynomial functions [6]. These functions are not yet appropriate as finite/boundary elements as they do not naturally enforce continuity of trace at element boundaries. This is addressed in section 3 where a geometric decomposition is developed which results in appropriate DOF defined in terms of test forms which span sub-spaces of the canonical function space. The construction of bases then becomes largely a matter of the choice of a basis for these test forms. We construct hierarchical spaces of test forms which lead to an explicit projection operator, written in terms of the DOF. Inversion of the projection operator leads naturally to appropriate hierarchical bases. Finally, explicit examples up to 2nd or 3rd order for all elements considered are tabulated in section 4 together with an analysis of condition number growth of the mass, quasi-stiffness and composite matrices.

\section{Construction of Canonical Spaces}

As is increasingly common, we shall use the calculus of differential forms to describe this approach. It is not an aim of this paper to provide an introduction to these methods; there are numerous, more instructive sources available (eg. [23]). However, we will introduce some relevant basic properties, so as to fix our notation.

\subsection{Properties of differential forms}

A differential form of order $r$ on a manifold $M$ on dimension $m$ is a totally anti-symmetric tensor of type $(0, r)$. That is, they are entirely covariant. We have the following general 
definition:

If we denote the vector space of $r$-forms at a point $p \in M$ by $\Omega_{p}^{r}(M)$, an element $\omega^{r} \in$ $\Omega_{p}^{r}(M)$ is expanded as

$$
\omega^{r}=\frac{1}{r !} \omega_{\mu_{1} \mu_{2} \cdots \mu_{r}}^{r} d x^{\mu_{1}} \wedge d x^{\mu_{2}} \wedge \cdots \wedge d x^{\mu_{r}}
$$

Note that for consistency with the above definitions, we define 0 -forms to be the space of real functions. When no confusion can arise we will usually drop the index ' $r$ '.

\subsubsection{Exterior derivative}

The exterior derivative $d$ is a map $d: \Omega^{r}(M) \longrightarrow \Omega^{r+1}(M)$ whose action on an $r$-form $\omega$ is defined by

$$
d \omega=\frac{1}{r !}\left(\frac{\partial}{\partial x^{v}} \omega_{\mu_{1} \cdots \mu_{r}}\right) d x^{v} \wedge d x^{\mu_{1}} \wedge \cdots \wedge d x^{\mu_{r}}
$$

What follows is an important formula, which is easy to prove from the basic definitions:

$$
d^{2} \omega=d(d \omega)=0
$$

The following Leibnitz formula can also be obtained:

$$
d\left(\omega^{q} \wedge \eta^{r}\right)=\left(d \omega^{q}\right) \wedge \eta^{r}+(-1)^{q} \omega^{q} \wedge\left(d \eta^{r}\right)
$$

There are two subspaces of $\Omega^{r}(M)$ which will be central to our subsequent analysis: The space of $r$-forms whose exterior derivatives vanish are denoted by $Z^{r}(M)$ and is often termed the null space of $r$-forms. Any $\omega^{r} \in Z^{r}(M)$ is said to be closed. The space of $r$-forms which are the exterior derivative of some $(r-1)$-form $\in \Omega^{r-1}(M)$ is denoted by $B^{r}(M)$. Any $\omega^{r} \in B^{r}(M)$ is said to be exact and $\exists \mu^{r-1} \in \Omega^{r-1}(M)$ such that $\omega^{r}=d \mu^{r-1}$. Equivalently, $B^{r}(M)=d \Omega^{r-1}(M)=\operatorname{Im}(d)$. Using (2.3) it is clear that $B^{r}(M) \subset Z^{r}(M)$, and we can then define so-called de Rham cohomology groups as the quotient $H^{r}(M)=Z^{r}(M) / B^{r}(M)$. The relevance of these cohomology groups is that for the $C^{\infty}$ case (ie, non-discrete) they are topological invariants. This property follows from the duality between these groups and homology groups, expressed by de Rham's theorem ( [23]):

Theorem 2.1. (de Rham's theorem): If $M$, is a compact manifold, $H_{r}(M)$ and $H^{r}(M)$ are finitedimensional, then the map $\Lambda: H_{r}(M) \times H^{r}(M) \rightarrow \mathbb{R}$ defined by

$$
\Lambda([c],[\omega])=\int_{c} \omega
$$

is bilinear and non-degenerate. Thus $H_{r}(M)$ is the dual vector space of $H^{r}(M)$.

Essentially, it is this feature of the continuum (the duality of homology and cohomology) which we seek to mimic in the discrete case. An important result for the subsequent analysis is that for any contractible manifold $M$ :

$$
H^{r}(M)=\left\{\begin{array}{l}
\mathbb{R} \text { if } r=0 \\
\varnothing \text { if } r>0
\end{array}\right.
$$




\subsubsection{Pull-back of differential forms}

Consider a map $f: M \longrightarrow N$ between two manifolds $M$ and $N$. This map also results in a corresponding map between tangent spaces in the manifolds, namely a push-forward:

$$
f_{*}: T_{p} M \rightarrow T_{f(p)} N
$$

Differential forms, however, which are operators on tangent spaces, are then subject to a corresponding map $f_{*}$, called the pull-back, ie

$$
f^{*}: \Omega^{*}(N) \rightarrow \Omega^{*}(M)
$$

Note that this map acts in the opposite sense as $f$ and $f_{*}$. It is also important to note that the exterior derivative commutes with the pull-back, ie.

$$
d f^{*} \omega=f^{*} d \omega
$$

In our applications we will usually consider $f$ to be a map from an intrinsic space specified on a reference element to a manifold in an appropriate element in physical space. This is usually done via interpolatory shape functions. The pull-back then takes differential forms from our physical element onto differential forms on the reference element.

\subsubsection{Trace and Continuity}

The trace of a differential form is essentially the restriction of a form defined on a manifold $M$ to a sub-manifold $\Gamma \subset M$ and is written as $\left.\omega^{r}\right|_{\Gamma}$ or $\operatorname{tr}_{\Gamma}\left(\omega^{r}\right)$. This is an important example of the application of the pull-back. If $\Gamma \subset M$ then we may always define a map $f: \Gamma \rightarrow M$ and we can define the trace as

$$
\operatorname{tr}_{\Gamma}\left(\omega^{r}\right)=f^{*} \omega^{r}
$$

It can be proven that the above expression is itself an $r$-form on $\Gamma \subset M$. Note also that the trace then commutes with the exterior derivative. Let us now consider a partitioning of a manifold $M$ into disjoint manifolds $M_{1}$ and $M_{2}$ with a common boundary $\Gamma$. Define an $r$-form on each of $M_{1}$ and $M_{2}$ by $\alpha^{r}$ and $\beta^{r}$ respectively. Then the two forms agree on $\Gamma$ if

$$
\operatorname{tr}_{\Gamma}\left(\alpha^{r}\right)=\operatorname{tr}_{\Gamma}\left(\beta^{r}\right)
$$

A useful result is that the trace is distributive over the exterior product, ie.

$$
\operatorname{tr}_{\Gamma}(\alpha \wedge \beta)=\operatorname{tr}_{\Gamma}(\alpha) \wedge \operatorname{tr}_{\Gamma}(\beta)
$$

Differential forms naturally possess the degrees of continuity that are required by real physical quantities such as electric fields (1-forms) and magnetic fluxes (2-forms). The apparent mixture of continuity requirements is simply a misleading result of the usual vector calculus, which defines continuity in terms of individual vector components. This problem is elegantly resolved by use of differential forms, as their continuity is defined in terms of their operations as mappings on tangent vectors. 


\subsubsection{Inner Product}

Note that two $r$-forms $\alpha$ and $\beta$ can always be combined to give an $m$-form $\alpha \wedge * \beta$, where $*$ is a well-defined Hodge operator. The integral of $\alpha \wedge * \beta$ over $M$ is then well defined. We thus define an inner product of two $r$-forms by,

$$
(\alpha, \beta)=\int \alpha \wedge * \beta
$$

Since $\alpha \wedge * \beta=\beta \wedge * \alpha$, the inner product is symmetric, ie.

$$
(\alpha, \beta)=(\beta, \alpha)
$$

Any two $r$-forms $\alpha$ and $\beta$ for which $(\alpha, \beta)=0$ are said to be orthogonal. It is important to note that we can form appropriate Hilbert spaces using this inner product. Using Stokes theorem, the following useful identity may be derived ( [23]):

$$
(d \alpha, \beta)=\left(\alpha, d^{\dagger} \beta\right)+\int_{\partial M} \alpha \wedge * \beta
$$

\subsection{Canonical Spaces}

We begin by considering an arbitrary $m$-dimensional element $M^{\prime} \in \mathbb{R}^{m}$ on which we have spaces of differential forms $\Omega^{r}\left(M^{\prime}\right)$. We will assume that there exists a parent element $M$ and a well-defined map $f: M \rightarrow M^{\prime}$. These parent elements are the usual base simplices, cartesian product spaces, prisms and pyramids on which appropriate intrinsic coordinates are defined. For any $\omega^{\prime} \in \Omega^{r}\left(M^{\prime}\right)$ we have, by pull-back, a corresponding $\omega \in \Omega^{r}(M)$. In this way, our analysis is applicable to general curvilinear elements.

Throughout this work, we will be concerned with polynomial differential forms $\omega \in$ $\Omega^{r}(M)$. These are forms where $\omega_{\mu_{1} \mu_{2} \cdots \mu_{r}}^{r}$ in (2.1) is a polynomial in the intrinsic coordinates on $M$. Before we move onto particular cases, it is useful to begin with a general discussion on the form that such spaces take. Given any linear operator (in this case the exterior derivative), we can clearly decompose any finite dimensional (ie. Discrete, hence the $h$ subscript) $\Omega_{h}^{r}(M)$ into its null subspace and its complement as follows

$$
\Omega_{h}^{r}(M)=Z_{h}^{r}(M) \oplus\left(Z_{h}^{r}(M)\right)^{c}
$$

where the superscript $c$ in (2.15) represents the complement. It is a basic result of linear algebra that $\left(Z_{h}^{r}(M)\right)^{c} \cong \operatorname{Im}(d)=B_{h}^{r+1}(M)$ (as described in Section 2.1.1), where $\cong$ indicates isomorphism. An isomorphism is a map which is bijective (1-1 and onto, hence an inverse exists). Note it is not necessary for the elements of the spaces to be of the same kind, merely that for each element in one space there is one (and only one) element in the other. We can, as with $C^{\infty}$ differential forms, formally compute cohomology groups. There is no guarantee, however, that these satisfy de Rham's theorem, ie. that they are 
dual to the underlying homology. Indeed, they may well not even be topological invariants at all. We must then ensure that this is the case, and it is this requirement which motivates the next step: If the $(r+1)^{t h}$-cohomology group is to be trivial (as it is for all of the cases discussed here, ie. contractible manifolds), then we see immediately that

$$
\left(Z_{h}^{r}(M)\right)^{c} \cong Z_{h}^{r+1}(M)
$$

This means that there must exist an isomorphism $\kappa$ such that

$$
\Omega_{h}^{r}(M)=Z_{h}^{r}(M) \oplus \kappa\left(Z_{h}^{r+1}(M)\right)
$$

It is important to note that this construction is invariant under pull-back, and certainly does not impose any restriction to affine maps on $M$. The isomorphism $\kappa$ is certainly not unique. Note that this isomorphism is called a lifting operator by [18], where the Poincaré map is used to construct Whitney elements on simplices. More recently the Koszul operator has been used in this role [4] and it is this approach we take here. Specifically, for any $r$-form $\omega$ defined as in (2.1), the action of Koszul operator is defined as

$$
\kappa \omega^{r}=\frac{1}{r !} \sum_{i=1}^{r}(-1)^{i+1} \omega_{\mu_{1} \cdots \mu_{i} \cdots \mu_{r}}^{r} x^{\mu_{i}} d x^{\mu_{1}} \wedge \cdots \widehat{d x^{\mu_{i}}} \wedge \cdots \wedge d x^{\mu_{r}}
$$

where the $\widehat{d x^{\mu_{i}}}$ term is omitted.

One final important point is to verify that these spaces are comprised of linearly independent sets of forms, for if this is not the case the essential property of unisolvency will not hold. This issue is resolved in the following theorem:

Theorem 2.2. If the elements of the spaces $\Omega_{h}^{r-1}(M)$ and $Z_{h}^{r+1}(M)$ are linearly independent, then the elements of $\Omega_{h}^{r}(M)=Z_{h}^{r}(M) \oplus \kappa\left(Z_{h}^{r+1}(M)\right)$ are also linearly independent.

Proof. Noting that $Z_{h}^{r}(M)=d \Omega_{h}^{r-1}(M)$ let us assume that there are elements of $Z_{h}^{r}(M)$ which are are linearly dependent. That is, $\exists \alpha_{i} \in \Omega_{h}^{r-1}(M)$ such that $d \alpha_{1}=\sum_{i=2}^{N_{1}} b_{i} d \alpha_{i}$ where $N_{1}=\operatorname{dim} \Omega_{h}^{r-1}(M)-1$ and $b_{i}$ are constants, not all zero. Thus $d\left(\alpha_{1}-\sum_{i=2}^{N_{1}} b_{i} \alpha_{i}\right)=0 \Longrightarrow \alpha_{1}-$ $\sum_{i=2}^{N_{1}} b_{i} \alpha_{i}=k$ for some constant $k$. Hence $\alpha_{1}=\sum_{i=2}^{N_{1}} b_{i} \alpha_{i}+k$ and then $\Omega_{h}^{r-1}(M)$ is not linearly independent. This is a contradiction and as a result $Z_{h}^{r}(M)$ is linearly independent.

We now apply a similar argument to the space $\kappa\left(Z_{h}^{r+1}(M)\right)$ : Let us assume that $\kappa\left(Z_{h}^{r+1}(M)\right)$ is linearly dependent. That is, $\exists \omega_{i} \in Z_{h}^{r+1}(M)$ such that $\kappa\left(\omega_{1}\right)=\sum_{j=2}^{N_{2}} c_{j} \kappa\left(\omega_{1}\right)$ where $N_{2}=\operatorname{dim} Z_{h}^{r+1}(M)$ and $c_{i}$ are constants, not all zero. Thus from the linearity of $\kappa$, we have $\kappa\left(\omega_{1}-\sum_{j=2}^{N_{2}} c_{j} \omega_{j}\right)=0 \Longrightarrow \omega_{1}-\sum_{j=2}^{N_{2}} c_{j} \omega_{j}=0$. Hence $\omega_{1}=\sum_{i=2}^{N} c_{j} \omega_{j}$ and then $Z_{h}^{r+1}(M)$ is not linearly independent. This is again a contradiction and as a result $\kappa\left(Z_{h}^{r+1}(M)\right)$ must be linearly independent. 
It remains to show that taken together, the spaces $Z_{h}^{r}(M)$ and $\kappa\left(Z_{h}^{r+1}(M)\right)$ are mutually independent: If this is not the case, then $\exists b_{i}, c_{j}, \alpha_{i}, \omega_{j}$ such that $d\left(\sum_{i=2}^{N_{1}} b_{i} \alpha_{i}\right)+\kappa\left(\sum_{j=2}^{N_{2}} c_{j} \omega_{j}\right)=$ 0 where not all of the $b_{i}$ and $c_{j}$ are zero. Applying the exterior derivative to both sides gives $d \kappa\left(\sum_{j=2}^{N_{2}} c_{j} \omega_{j}\right)=0 \Longrightarrow\left(\sum_{j=2}^{N_{2}} c_{j} \omega_{j}\right)=0$ which implies that $Z_{h}^{r+1}(M)$ is not linearly independent. Again we have a contradiction and the proof is complete.

\subsection{Simplexes}

The results on simplices are specific realisations of the results given in [4] and are presented here for completeness and to aid the subsequent development for other element geometries. Before we proceed, it is useful to define some polynomial spaces which we will use throughout the following discussion. We denote the complete space of polynomials of order $\leq k$ on a simplex $M$ in $n$-dimensions as $P_{n, k}(M)$ and the space of homogeneous polynomials of order $=k$ as $\tilde{P}_{n, k}(M)$. We will frequently remove the dependence on $M$ where no confusion arises.

The dimension of these polynomial spaces is given by

$$
\operatorname{dim} P_{n, k}=\left(\begin{array}{c}
n+k \\
k
\end{array}\right), \operatorname{dim} \tilde{P}_{n, k}=\left(\begin{array}{c}
n+k-1 \\
k
\end{array}\right)
$$

\subsubsection{Two dimensional case.}

In two space dimensions we define a graded algebra of polynomial differential forms as follows: We select a polynomial space of 0 -forms, complete to order $k_{0}$. Thus we have

$$
\Omega_{k_{0}}^{0}=P_{2, k_{0}}=\left\{\zeta_{1}^{l} \zeta_{2}^{m} ; l+m \leq k_{0}\right\}
$$

Similarly we also define a complete space of 2 -forms, complete to order $k_{2}$, so we have

$$
\Omega_{k_{2}}^{2}=P_{2, k_{2}} d \zeta_{1} \wedge d \zeta_{2}
$$

Note that the exterior derivative of all 2-forms vanish in two dimensions so this space is also the null space of 2-forms, ie $Z_{k_{2}}^{2}=\Omega_{k_{2}}^{2}$. We then have a graded algebra defined by

$$
\Omega^{*}=\Omega_{k_{0}}^{0} \oplus \Omega_{k_{0} k_{2}}^{1} \oplus \Omega_{k_{2}}^{2}
$$

where $\Omega_{k_{0}}^{0}=P_{2, k_{0}}, \Omega_{k_{0} k_{2}}^{1}=Z_{k_{0}}^{1} \oplus \kappa\left(\Omega_{k_{2}}^{2}\right)$ and $\Omega_{k_{2}}^{2}=P_{2, k_{2}} d \zeta_{1} \wedge d \zeta_{2}$.

Note that $Z_{k_{0}}^{1}$ is uniquely defined by $\Omega_{k_{0}}^{0}$ since $Z_{k_{0}}^{1}=d \Omega_{k_{0}}^{0}$. Hence, using (2.20) we have

$$
Z_{k_{0}}^{1}=\bigoplus_{j=1 l+m=j}^{k_{0}} \oplus\left(l \zeta_{1}^{l-1} \zeta_{2}^{m} d \zeta_{1}+m \zeta_{1}^{l} \zeta_{2}^{m-1} d \zeta_{2}\right)
$$


Applying the Koszul operator to (2.21) then gives:

$$
\kappa\left(\Omega_{k_{2}}^{2}\right)=\bigoplus_{j=0 l+m=j}^{k_{2}} \zeta_{1}^{l} \zeta_{2}^{m}\left(\zeta_{2} d \zeta_{1}-\zeta_{1} d \zeta_{2}\right)
$$

We can easily compute the dimensions of the spaces of differential forms since $\operatorname{dim} Z_{k_{0}}^{1}=$ $\operatorname{dim} P_{2, k_{0}}-1$, and the dimension of a space is preserved under isomorphism, hence:

$$
\begin{aligned}
\operatorname{dim} \Omega_{k_{0}}^{0} & =\frac{1}{2}\left(k_{0}+1\right)\left(k_{0}+2\right) \\
\operatorname{dim} \Omega_{k_{0} k_{2}}^{1} & =\frac{1}{2}\left(k_{0}+1\right)\left(k_{0}+2\right)+\frac{1}{2}\left(k_{2}+1\right)\left(k_{2}+2\right)-1 \\
\operatorname{dim} \Omega_{k_{2}}^{2} & =\frac{1}{2}\left(k_{2}+1\right)\left(k_{2}+2\right)
\end{aligned}
$$

We have thus obtained all of the polynomial differential forms needed to define the graded algebra (2.22): These 0 -form and 2-form spaces are complete (and linearly independent) up to orders $k_{0}$ and $k_{2}$ respectively. As a result of this and theorem 2.2 we see that $\Omega_{k_{0} k_{2}}^{1}$ is linearly independent. It is desirable (though not necessary) for the 1 -form spaces to be complete up to some degree: Firstly this provides us with homogeneous spaces of forms whose orders of approximation are identical along all edges. Secondly, such a feature is useful in the confirmation of commuting diagram properties, (although it is the opinion of the author that such properties are to be viewed as sufficient, rather than necessary conditions). It can easily be shown that the 1-form spaces $\Omega_{k_{0} k_{2}}^{1}$ are complete up to order $\min \left(k_{0}-1, k_{2}+1\right)$. Equivalently, then, for $\Omega_{k_{0} k_{2}}^{1}$ to be complete up to order $k_{0}-1$, we must have $k_{2} \geq k_{0}-2$. In section 3.6, the satisfaction of commuting diagram properties will require that

$$
d^{\dagger} \Omega_{k_{0} k_{2}}^{1} \subset \Omega_{k_{0}}^{0} \text { and } d^{\dagger} \Omega_{k_{2}}^{2} \subset \Omega_{k_{0} k_{2}}^{1}
$$

where the adjoint exterior derivative is based on a trivial intrinsic metric.

These provide a further (sufficient) constraint on $k_{2}$ as follows: Since the $\Omega_{k_{0} k_{2}}^{1}$ are polynomial 2-forms of degree at most $\max \left(k_{0}-1, k_{2}+1\right)$ then from the first expression in (2.25) we would require $k_{2}+1 \leq k_{0} \Longleftrightarrow k_{2}<k_{0}$. Consideration of the second expression in (2.25) leads to a similar constraint (strictly speaking, it leads to $k_{2} \leq k_{0}$ but this is weaker than the first). Summarizing, then, in such circumstances we would require $k_{0}>k_{2} \geq k_{0}-2$, that is, we have either $k_{2}=k_{0}-1$ or $k_{2}=k_{0}-2$.

If we choose $k_{2}=k_{0}-1$, then the dimension of the 1-form spaces reduce to the expressions derived by [16] and result in spaces analogous to Nédélecs first family. Alternatively choosing $k_{2}=k_{0}-2$ results in Nédélecs second family. Note that choices outside these limits can be made, but will result in inhomogeneous spaces with differing orders of approximation on each edge, as has been demonstrated in [5]. Homogeneous spaces 
arise only when $k_{2}=k_{0}-1$ or $k_{2}=k_{0}-2$. Generally, then, for any given order $k$ we have two homogeneous graded algebras, given by

$$
\Omega_{k}^{*}=\Omega_{k}^{0} \oplus\left\{\begin{array}{l}
\Omega_{k(k-1)}^{1} \oplus \Omega_{k-1}^{2} \\
\Omega_{k(k-2)}^{1} \oplus \Omega_{k-2}^{2}
\end{array}\right.
$$

Implementations of this two dimensional case are given in [5] in the context of boundary elements and we refer the reader to that article for more specific details.

\subsubsection{Three dimensional case.}

In three space dimensions we follow a process largely identical to the two dimensional case: Firstly we select a polynomial space of 0 -forms, complete to order $k_{0}$. Thus we have

$$
\Omega_{k_{0}}^{0}=P_{3, k_{0}}=\left\{\zeta_{1}^{l} \zeta_{2}^{m} \zeta_{3}^{n} ; l+m+n \leq k_{0}\right\}
$$

We then define a complete space of 3-forms, complete to order $k_{3}$, so we have

$$
\Omega_{k_{3}}^{3}=P_{3, k_{3}} d \zeta_{1} \wedge d \zeta_{2} \wedge d \zeta_{3}
$$

Note that now the exterior derivative of all 3-forms vanish so this space is also the null space of 3-forms, ie $Z_{k_{3}}^{3}=\Omega_{k_{3}}^{3}$. We then have a tentative graded algebra defined by

$$
\Omega^{*}=\Omega_{k_{0}}^{0} \oplus \Omega_{k_{0} k_{2}}^{1} \oplus \Omega_{k_{2} k_{3}}^{2} \oplus \Omega_{k_{3}}^{3}
$$

where $\Omega_{k_{0} k_{2}}^{1}=Z_{k_{0}}^{1} \oplus \kappa\left(Z_{k_{2}}^{2}\right)$ and $\Omega_{k_{2} k_{3}}^{2}=Z_{k_{2}}^{2} \oplus \kappa\left(\Omega_{k_{3}}^{3}\right)$.

Note again that $Z_{k_{0}}^{1}$ is uniquely defined by $\Omega_{k_{0}}^{0}$ since $Z_{k_{0}}^{1}=d \Omega_{k_{0}}^{0}$. Hence, from (2.27) we have

$$
Z_{k_{0}}^{1}=\bigoplus_{j=1 l+m+n=j}^{k_{0}} \oplus_{1}\left(l \zeta_{1}^{l-1} \zeta_{2}^{m} \zeta_{3}^{n} d \zeta_{1}+m \zeta_{1}^{l} \zeta_{2}^{m-1} \zeta_{3}^{n} d \zeta_{2}+n \zeta_{1}^{l} \zeta_{2}^{m} \zeta_{3}^{n-1} d \zeta_{3}\right)
$$

For the next step to complete the definition of the graded algebra we need to define $Z_{k_{2}}^{2}$. Unlike the definitions of $Z_{k_{0}}^{1}$ and $Z_{k_{3}}^{3}$, this is a non-trivial activity. To obtain such a space we define a prototype space $Z_{p, k_{2}}^{2}$ as the space of 2-forms with coefficients which are themselves complete spaces of polynomials up to order $k_{2}$. Furthermore we can write $Z_{p, k_{2}}^{2}$ as the direct sum of homogeneous null spaces $\tilde{Z}_{p, k}^{2}$ of orders up to and including $k_{2}$. Hence

$$
Z_{p, k_{2}}^{2}=\tilde{Z}_{p, 0}^{2} \oplus \tilde{Z}_{p, 1}^{2} \oplus \cdots \oplus \tilde{Z}_{p, k_{2}}^{2}
$$

These spaces have dimension given by

$$
\operatorname{dim} \tilde{Z}_{p, k_{2}}^{2}=3\left(\begin{array}{c}
2+k_{2} \\
k_{2}
\end{array}\right)
$$




$$
\operatorname{dim} Z_{p, k_{2}}^{2}=3\left(\begin{array}{c}
3+k_{2} \\
k_{2}
\end{array}\right)
$$

We now need to reduce the dimension of this space by selecting only those forms which vanish under the application of the exterior derivative. For each of the prototype homogeneous null spaces $\tilde{Z}_{p, k}^{2}$ the action of the exterior derivative will produce a homogeneous polynomial 3-form of degree $k-1$. We can create an incidence matrix $M$ where the rows are identified with the members of $\tilde{Z}_{p, k}^{2}$ and the columns are identified with 3-forms $\tilde{\Omega}_{k-1}^{3}$ whose coefficients are members of $\tilde{P}_{3, k-1}$. The entries correspond to the action of the exterior derivative on each of the members of $\tilde{Z}_{p, k}^{2}$. We can construct an augmented matrix, $A M$, by appending the identity matrix of order $\operatorname{dim} \tilde{Z}_{p, k}^{2}$ to $M$, giving $A M=[M \mid I]$

The next step is to perform a row reduction of $M$ on the matrix $A M$, giving a matrix $A M_{\text {red }}=\left[M_{\text {red }} \mid N\right]$. We can then construct a matrix $N_{\text {red }}$ by removing any of the rows from $N$ for which $M_{\text {red }}$ is not rank deficient. The null space $\tilde{Z}_{p, k}^{2}$ is then given by

$$
\tilde{Z}_{k}^{2}=\left[N_{r e d}\right] \tilde{Z}_{p, k}^{2}
$$

We can repeat this process for every order $k=0, \cdots, k_{2}$, and construct the 2 -form null space from

$$
Z_{k_{2}}^{2}=\tilde{Z}_{0}^{2} \oplus \tilde{Z}_{1}^{2} \oplus \cdots \oplus \tilde{Z}_{k_{2}}^{2}
$$

Note that for each order $k$, the number of terms of order $k$ in the prototype space $\tilde{Z}_{p, k}^{2}$ is $3 \operatorname{dim} \tilde{P}_{3, k}$. The rank of the incidence matrix $M$ is precisely the dimension of the homogeneous polynomial space of degree $k-1$, that is $\operatorname{dim} \tilde{P}_{3, k-1}$. Note that by this construction, $Z_{k_{2}}^{2}$ is linearly independent. The dimension of this space can be computed as

$$
\operatorname{dim} \tilde{Z}_{k_{2}}^{2}=3 \sum_{k=0}^{k_{2}} \operatorname{dim} \tilde{P}_{3, k}-\sum_{k=0}^{k_{2}-1} \operatorname{dim} \tilde{P}_{3, k}=3 \operatorname{dim} \tilde{P}_{3, k_{2}}+2 \sum_{k=0}^{k_{2}-1} \operatorname{dim} \tilde{P}_{3, k}=3\left(\begin{array}{c}
2+k_{2} \\
k_{2}
\end{array}\right)+2\left(\begin{array}{c}
2+k_{2} \\
k_{2}-1
\end{array}\right)
$$

Using the above expression and (2.35), and with a little algebra, one can show that

$$
\operatorname{dim} Z_{k_{2}}^{2}=\frac{1}{6}\left(k_{2}+1\right)\left(k_{2}+2\right)\left(2 k_{2}+9\right)
$$

In fact this process results in the following closed form expression for the 2-form null 
spaces:

$$
\begin{aligned}
& Z_{k_{2}}^{2}=\bigoplus_{j=0}^{k_{2}}\left\{\underset{m+n=j}{\oplus} d \zeta_{2}^{m+1} \wedge d \zeta_{3}^{n+1} \oplus \underset{l+n=j}{\oplus} d \zeta_{3}^{n+1} \wedge d \zeta_{1}^{l+1} \oplus_{l+m=j}^{\oplus} d \zeta_{1}^{l+1} \wedge d \zeta_{2}^{m+1}\right\}
\end{aligned}
$$

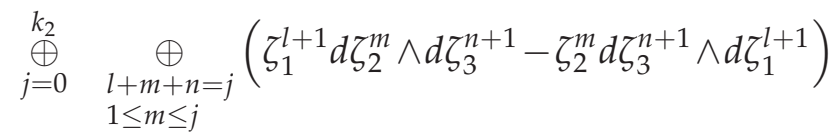

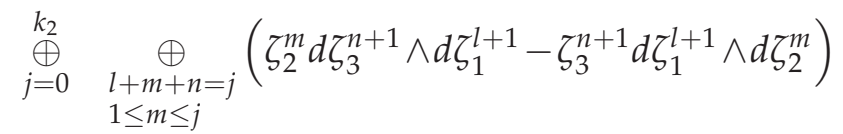

Applying the Koszul operator then gives

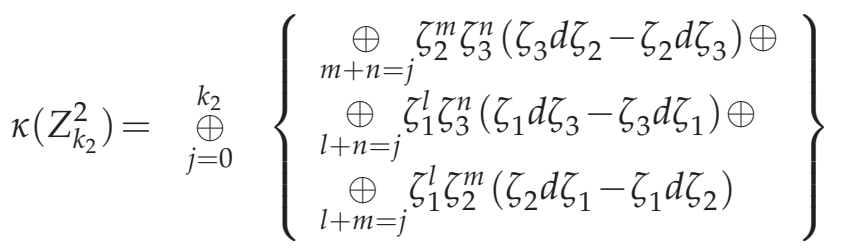

$$
\begin{aligned}
& \bigoplus_{j=0}^{k_{2}} \underset{\substack{l+m+n=j \\
1 \leq m \leq j}}{\oplus}\left(\begin{array}{c}
m(n+1) \zeta_{1}^{l+1} \zeta_{2}^{m-1} \zeta_{3}^{n}\left(\zeta_{3} d \zeta_{2}-\zeta_{2} d \zeta_{3}\right) \\
-(l+1)(n+1) \zeta_{1}^{l} \zeta_{2}^{m} \zeta_{3}^{n}\left(\zeta_{1} d \zeta_{3}-\zeta_{3} d \zeta_{1}\right)
\end{array}\right) \\
& \bigoplus_{j=0}^{k_{2}} \underset{\substack{l+m+n=j \\
1 \leq m \leq j}}{\bigoplus}\left(\begin{array}{c}
(l+1)(n+1) \zeta_{1}^{l} \zeta_{2}^{m} \zeta_{3}^{n}\left(\zeta_{1} d \zeta_{3}-\zeta_{3} d \zeta_{1}\right) \\
-(l+1) m \zeta_{1}^{l} \zeta_{2}^{m-1} \zeta_{3}^{n+1}\left(\zeta_{2} d \zeta_{1}-\zeta_{1} d \zeta_{2}\right)
\end{array}\right)
\end{aligned}
$$

Finally, $\kappa\left(\Omega_{k_{3}}^{3}\right)$ is readily obtained by application of (2.18) to (2.28):

$$
\kappa\left(\Omega_{k_{3}}^{3}\right)=\bigoplus_{j=0 l+m+n=j}^{k_{3}} \zeta_{1}^{l} \zeta_{2}^{m} \zeta_{3}^{n}\left(\zeta_{1} d \zeta_{2} \wedge d \zeta_{3}+\zeta_{2} d \zeta_{3} \wedge d \zeta_{1}+\zeta_{3} d \zeta_{1} \wedge d \zeta_{2}\right)
$$

All of the required elements of the graded algebra (2.29) are thus obtained. Again, we can easily compute the dimensions of the spaces of differential forms since $\operatorname{dim} Z_{k_{0}}^{1}=$ $\operatorname{dim} P_{3, k_{0}}-1$, and the dimension of a space is preserved under isomorphism, hence:

$$
\begin{aligned}
\operatorname{dim} \Omega_{k_{0}}^{0} & =\frac{1}{6}\left(k_{0}+1\right)\left(k_{0}+2\right)\left(k_{0}+3\right) \\
\operatorname{dim} \Omega_{k_{0} k_{2}}^{1} & =\frac{1}{6}\left(k_{0}+1\right)\left(k_{0}+2\right)\left(k_{0}+3\right)+\frac{1}{6}\left(k_{2}+1\right)\left(k_{2}+2\right)\left(2 k_{2}+9\right)-1 \\
\operatorname{dim} \Omega_{k_{2} k_{3}}^{2} & =\frac{1}{6}\left(k_{2}+1\right)\left(k_{2}+2\right)\left(2 k_{2}+9\right)+\frac{1}{6}\left(k_{3}+1\right)\left(k_{3}+2\right)\left(k_{3}+3\right) \\
\operatorname{dim} \Omega_{k_{3}}^{3} & =\frac{1}{6}\left(k_{3}+1\right)\left(k_{3}+2\right)\left(k_{3}+3\right)
\end{aligned}
$$


Using theorem 2.2 and the linear independence of $\Omega_{k_{0}}^{0}$ and $Z_{k_{2}}^{2}$ we see that $\Omega_{k_{0} k_{2}}^{1}$ is linearly independent. In the same way the linear independence of $\Omega_{k_{2} k_{3}}^{2}$ is ensured by that of $\Omega_{k_{0} k_{2}}^{1}$ and $\Omega_{k_{3}}^{3}$. Constraints on $k_{0}, k_{2}$ and $k_{3}$ can be arrived at in an identical manner to that in section 2.3.1 for two dimensional simplexes. The 0 -forms and 3-forms are complete to orders $k_{0}$ and $k_{3}$ respectively; 1 -forms are complete to order $\min \left(k_{0}-1, k_{2}+1\right)$ and 2-forms are complete to order $\min \left(k_{2}, k_{3}+1\right)$. The desire for homogeneous spaces and commuting properties leads to an identical set of constraints for $k_{0}$ and $k_{2}$, namely $k_{0}>k_{2} \geq k_{0}-2$. The corresponding analysis for 2 and 3 forms leads to the additional constraint $k_{2} \geq k_{3} \geq k_{2}-1$. These guarantee that the following properties are satisfied:

$$
d^{\dagger} \Omega_{k_{0} k_{2}}^{1} \subset \Omega_{k_{0}}^{0} d^{\dagger} \Omega_{k_{2} k_{3}}^{2} \subset \Omega_{k_{0} k_{2}}^{1} \text { and } d^{\dagger} \Omega_{k_{3}}^{2} \subset \Omega_{k_{2} k_{3}}^{2}
$$

As a result, in such circumstances, for any given order $k$ we have four possible graded algebras, given by

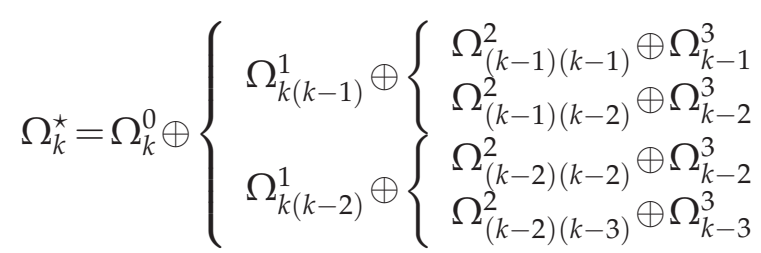

Note that from (2.26) and (2.43) we see that on simplexes there are two distinct graded algebras of order $k$ in two dimensions and four in three dimensions which is entirely consistent with the results in [4]. We can relate these algebras to the classification in [15]: For 1-forms (curl conforming), B and C-type spaces correspond to $\Omega_{k(k-1)}^{1}$ and $\Omega_{k(k-2)}^{1}$ respectively. We can similarly extend this classification to 2-forms (div conforming), denoting $\Omega_{k k}^{2}$ and $\Omega_{k(k-1)}^{2}$ as B and C-type spaces respectively.

\subsection{Tensor product spaces}

The application of the Koszul operator is not the only method for constructing appropriate spaces. Notably, for tensor product spaces such as arise on squares and hexahedra we can use the Künneth formula ( [23]). Indeed, this approach has the clear advantage of not requiring computation of any null spaces. Let us first consider the one dimensional case: The fact that, geometrically speaking, a quadrilateral is the product of two line manifolds guides us in the construction of the basis forms. Let us define a simple one-dimensional manifold $M$, so $n=1$. Then we can define 0 -forms and 1 -forms in terms of univariate polynomials, so, $\Omega^{0}(M)=P_{1, k}\left(\zeta_{1}\right), \Omega^{1}(M)=P_{1, j}\left(\zeta_{1}\right) d \zeta_{1}$ where again $P_{k}\left(\zeta_{1}\right)$ is a polynomial of degree at most $k$. Up to this point we have not said anything regarding the degrees of these polynomials, indeed they can (and will) be different, as is shown in the subsequent analysis. The graded algebra associated with the above is then $\Omega^{*}(M)=\Omega^{0}(M) \oplus \Omega^{1}(M)$. The cohomology groups can be computed quite simply as follows: $Z^{0}(M)$ arises from the constant term in the polynomial $P_{k}\left(\zeta_{1}\right)$, so $Z^{0}(M)=\mathbb{R}$. Moreover, $B^{0}(M)=\varnothing$, since there 
are no (-1)-forms which implies that $H^{0}(M)=\mathbb{R}$. Now $Z^{1}(M)=\Omega^{1}(M) \cong \underset{k^{\prime}=0}{\bigoplus} \mathbb{R}$ since 2-forms do not exist in one dimension and $B^{1}(M)=d \Omega^{0}(M)=P_{1, k-1}\left(\zeta_{1}\right) d \zeta_{1} \cong \underset{k^{\prime}=0}{\oplus \rightarrow 1} \mathbb{R}$ thus $H^{1}(M)=\underset{k^{\prime}=0}{\dagger-k+1} \mathbb{R}$. The trivial requirement that $B^{1}(M) \subset Z^{1}(M)$ then implies that $j \geq k-1$.

As stated earlier, quadrilaterals (and hexahedra) $M$ are the product of simple onedimensional manifolds $M_{1}, M_{2}$ (and $M_{3}$ ) of the type discussed above. The corresponding graded algebra is then formed as a tensor product of the one-dimensional algebras. In $n$-dimensions this takes the form

$$
\begin{aligned}
\Omega^{*}(M) & =\underset{m=1}{\otimes}\left(\Omega_{i}^{0}\left(M_{m}\right) \oplus \Omega_{j}^{1}\left(M_{m}\right)\right) \\
& \equiv \underset{r=0}{\bigoplus} \Omega^{r}(M)
\end{aligned}
$$

where

$$
\Omega^{r}(M)=\underset{i_{1}+\cdots+i_{n}=r}{\oplus} \Omega^{i_{1}}\left(M_{1}\right) \otimes \cdots \otimes \Omega^{i_{n}}\left(M_{n}\right)
$$

We have still to address the fundamental issue of cohomology. The Künneth formula allows us to compute cohomology groups of $M$ as a function of the submanifolds $M_{i}$ as

$$
H^{r}(M)=\underset{i_{1}+\cdots+i_{n}=r}{\oplus} H^{i_{1}}\left(M_{1}\right) \otimes \cdots \otimes H^{i_{n}}\left(M_{n}\right)
$$

Using the Künneth formula, we can compute the cohomology groups of the manifold $M$ as follows:

$$
\begin{aligned}
& H^{0}(M)=\mathbb{R} \\
& H^{1}(M)=\left(\underset{k^{\prime}=0}{\oplus-k+1} \mathbb{R}\right) \otimes \mathbb{R} \otimes \cdots \otimes \mathbb{R}=\left\{\begin{array}{l}
\mathbb{R} \text { if } j>k-1 \\
\varnothing \text { if } j=k-1
\end{array}\right. \\
& H^{2}(M)=\left(\underset{k^{\prime}=0}{\oplus-k+1} \mathbb{R}\right) \otimes\left(\underset{k^{\prime}=0}{\oplus} \mathbb{R}\right) \otimes \cdots \otimes \mathbb{R}=\left\{\begin{array}{l}
\mathbb{R} \text { if } j>k-1 \\
\varnothing \text { if } j=k-1
\end{array}\right. \\
& \vdots \\
& H^{n}(M)=\left(\underset{k^{\prime}=0}{\oplus-k+1} \mathbb{R}\right) \otimes\left(\underset{k^{\prime}=0}{\oplus} \mathbb{R}\right) \otimes \cdots \otimes\left(\underset{k^{\prime}=0}{\oplus-k+1} \mathbb{R}\right)=\left\{\begin{array}{l}
\mathbb{R} \text { if } j>k-1 \\
\varnothing \text { if } j=k-1
\end{array}\right.
\end{aligned}
$$

Obviously, in order to preserve the continuous cohomology theory (that is, to maintain a trivial cohomology), we must choose polynomial dependencies satisfying $j=k-1$. In this case, the dimension of the conforming spaces is given by

$$
\operatorname{dim} \Omega^{r}(M)=\left(\begin{array}{l}
n \\
r
\end{array}\right)(k+1)^{n-r} k^{r}
$$


The final form of the canonical basis functions are then given by substituting $j=k-1$ into equation (2.45). For 1 -forms in two dimensions we readily obtain

$$
\omega=P_{1, k-1}\left(\zeta_{1}\right) P_{1, k}\left(\zeta_{2}\right) d \zeta_{1}+P_{1, k}\left(\zeta_{1}\right) P_{1, k-1}\left(\zeta_{2}\right) d \zeta_{2}
$$

and in three dimensions we have

$$
\begin{aligned}
\omega & =P_{1, k-1}\left(\zeta_{1}\right) P_{1, k}\left(\zeta_{2}\right) P_{1, k}\left(\zeta_{3}\right) d \zeta_{1} \\
& +P_{1, k}\left(\zeta_{1}\right) P_{1, k-1}\left(\zeta_{2}\right) P_{1, k}\left(\zeta_{3}\right) d \zeta_{2} \\
& +P_{1, k}\left(\zeta_{1}\right) P_{1, k}\left(\zeta_{2}\right) P_{1, k-1}\left(\zeta_{3}\right) d \zeta_{3}
\end{aligned}
$$

Expressions for other forms follow similarly. In the classification of Gragial et al. [15] these bases can be considered to be type B spaces.

\subsection{Prismatic elements}

Another common element type, essential for combining hexahedral and tetrahedral meshes, is the triangular prism. The construction of spaces on prisms is a combination of that for the $2 \mathrm{D}$ simplex outlined earlier and the tensor product spaces in section 2.4. The prism $M$ is considered to be a product of a line $L$ and a $2 \mathrm{D}$ simplex (triangle), $T$. The corresponding graded algebra is then formed as a tensor product of the one-dimensional line algebra and the two-dimensional simplex algebra. Hence

$$
\begin{aligned}
\Omega^{*}(M) & =\left(\Omega^{0}(L) \oplus \Omega^{1}(L)\right) \otimes\left(\Omega^{0}(T) \oplus \Omega^{1}(T) \oplus \Omega^{2}(T)\right) \\
& \equiv \Omega^{0}(M) \oplus \Omega^{1}(M) \oplus \Omega^{2}(M) \oplus \Omega^{3}(M)
\end{aligned}
$$

where

$$
\begin{aligned}
& \Omega^{0}(M)=\Omega^{0}(L) \otimes \Omega^{0}(T) \\
& \Omega^{1}(M)=\Omega^{0}(L) \otimes \Omega^{1}(T) \oplus \Omega^{1}(L) \otimes \Omega^{0}(T) \\
& \Omega^{2}(M)=\Omega^{0}(L) \otimes \Omega^{2}(T) \oplus \Omega^{1}(L) \otimes \Omega^{1}(T) \\
& \Omega^{3}(M)=\Omega^{1}(L) \otimes \Omega^{2}(T)
\end{aligned}
$$

As before, we have still to address the issue of cohomology. Using the Künneth formula, we can compute the cohomology groups of the prism $M$ as follows:

$$
\begin{aligned}
& H^{0}(M)=H^{0}(L) \otimes H^{0}(T) \\
& H^{1}(M)=H^{0}(L) \otimes H^{1}(T) \oplus H^{1}(L) \otimes H^{0}(T) \\
& H^{2}(M)=H^{1}(L) \otimes H^{1}(T) \oplus H^{0}(L) \otimes H^{2}(T) \\
& H^{3}(M)=H^{1}(L) \otimes H^{2}(T)
\end{aligned}
$$


Clearly, all we have to do is select the spaces $\Omega^{r}(L)$ and $\Omega^{r}(T)$ appropriately, so that we obtain the cohomology associated with a contractible manifold. Indeed, we can choose $\Omega^{r}(T)$ to be precisely those spaces defined in section 2.3.1. In that case, $H^{0}(T)=$ $\mathbb{R} ; H^{r}(T)=\varnothing, r>0$. The cohomology groups for the prism then reduce to

$$
H^{0}(M)=H^{0}(L) \otimes \mathbb{R} ; H^{1}(M)=H^{1}(L) \otimes \mathbb{R} ; H^{2}(M)=\varnothing ; H^{3}(M)=\varnothing
$$

For the line $L$ we can again choose spaces defined in section 2.4. We then have $H^{0}(L)=$ $\mathbb{R} ; H^{1}(L)=\underset{k^{\prime}=0}{\dagger-k+1} \mathbb{R}$. Clearly, unless we select $\Omega^{r}(L)$ such that $H^{0}(L)=\mathbb{R} ; H^{1}(L)=\varnothing$, we will fail to meet the necessary demands of topology. In short then, we must have $j=k-1$, just as we required in section 2.4. This results in the following function spaces:

$$
\begin{aligned}
\Omega_{k k_{0}}^{0}(M) & =\Omega_{k}^{0}(L) \otimes \Omega_{k_{0}}^{0}(T) \\
\Omega_{k k_{0} k_{2}}^{1}(M) & =\Omega_{k}^{0}(L) \otimes \Omega_{k_{0} k_{2}}^{1}(T) \oplus \Omega_{k-1}^{1}(L) \otimes \Omega_{k_{0}}^{0}(T) \\
\Omega_{k k_{0} k_{2}}^{2}(M) & =\Omega_{k}^{0}(L) \otimes \Omega_{k_{2}}^{2}(T) \oplus \Omega_{k-1}^{1}(L) \otimes \Omega_{k_{0} k_{2}}^{1}(T) \\
\Omega_{k k_{2}}^{3}(M) & =\Omega_{k-1}^{1}(L) \otimes \Omega_{k_{2}}^{2}(T)
\end{aligned}
$$

Influenced by the desire for homogeneous spaces and commuting diagrams we select $k$, $k_{0}$ and $k_{2}$ appropriately. This results in two possible graded algebras for a given order $k$ :

$$
\Omega_{k}^{*}=\Omega_{k k}^{0} \oplus\left\{\begin{array}{l}
\Omega_{k k(k-1)}^{1} \oplus \Omega_{k k(k-1)}^{2} \oplus \Omega_{k(k-1)}^{3} \\
\Omega_{k k(k-2)}^{1} \oplus \Omega_{k k(k-2)}^{2} \oplus \Omega_{k(k-2)}^{3}
\end{array}\right.
$$

Note that we can classify these 1 and 2-form bases as B and C-type depending on whether $\Omega^{1}(T)$ is type B or C.

\subsection{Pyramidal elements.}

Another case of great importance, particularly where both hexahedral, prismatic and simplicial elements are used in a mesh, are pyramidal elements, an example of which is depicted in Figure 1. As in the previous cases, we wish to construct a graded algebra of polynomial differential forms on this element. We begin by constructing, then, a space of 0-forms: However, we cannot expect this to be complete to a given order as in the case of a simplex. In fact, the construction of this 0 -form space is not the trivial activity it was for both simplexes and tensor product elements. Such spaces are normally obtained from hexahedral basis functions via an appropriate mapping. This results in spaces which are polynomials along edges and on faces, but are non-polynomial within the element. These spaces have been used to construct spaces of 1-forms [12]. More recently, piecewise wholly polynomial bases of scalars (0-forms) on pyramids have been developed [6] and it is this approach we employ here. The use of such piecewise polynomial spaces allows 


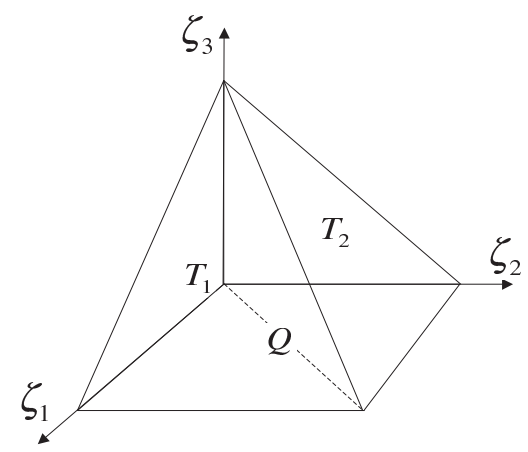

Figure 1: Pyramid (and constituent tetrahedra)

us to make use of the Koszul operator (applicable only to polynomial differential forms), as we did for simplicies.

To begin, we summarize the results in [6]: Consider the pyramid $M$ defined in Figure 1 where we have indicated two disjoint tetrahedra $T_{1}$ and $T_{2}$ which together form the pyramid and the square base $Q$. We then define the space of 0 -forms $\Omega_{k_{0}}^{0}(M)$ in terms of $\Omega_{k_{0}}^{0}\left(T_{3}\right)$ as

$$
\Omega_{k_{0}}^{0}(M)=\bigoplus_{k=0}^{k_{0}} \tilde{\Omega}_{k}^{0}(M)
$$

where

$$
\tilde{\Omega}_{k}^{0}(M)=\tilde{\Omega}_{k}^{0}\left(T_{3}\right) \oplus\left\{\tilde{P}_{4, k-1}\left(\zeta_{1}, \zeta_{2}, \zeta_{3}, \theta\right) \otimes \theta\right\}, \theta=\left\{\begin{array}{l}
\left(\zeta_{1}+\zeta_{3}\right) \zeta_{2} \\
\zeta_{1}\left(\zeta_{2}+\zeta_{3}\right)
\end{array}\right.
$$

To clarify the notation, $\theta$ corresponds to the upper expression on $T_{1}$ and the lower expression on $T_{2}$. The same interpretation applies to the use of braces in the rest of this work, unless otherwise stated. The dimension of these spaces can be computed as

$$
\operatorname{dim} \tilde{\Omega}_{k}^{0}(M)=\frac{1}{6}(k+1)(k+2)(k+3)
$$

Thus we have dimensions of $1,4,10$ and 20 , for $k=0,1,2$ and 3, and so on. The null space of 1 -forms is then readily computed from $Z_{k_{0}}^{1}=d \Omega_{k_{0}}^{0}$. Note we also have $\operatorname{dim} Z_{k_{0}}^{1}(M)=$ $\operatorname{dim} \Omega_{k_{0}}^{0}(M)-1$. We can define a space of 3-forms on $M$ using as for tetrahedra, ie.

$$
\Omega_{k_{3}}^{3}(M)=P_{3, k_{3}} d \zeta_{1} \wedge d \zeta_{2} \wedge d \zeta_{3}
$$

What remains is to compute the null space of 2 -forms $Z_{k_{2}}^{2}$. In fact we will propose the following general form for the null space:

$$
Z_{k_{2}}^{2}(M)=\bigoplus_{k=0}^{k_{2}} \tilde{Z}_{k}^{2}(M)
$$


where

$$
\begin{aligned}
& \tilde{Z}_{k}^{2}(M)=\tilde{Z}_{k}^{2}\left(T_{3}\right) \oplus \tilde{S}_{k}^{2}(M) \oplus \tilde{T}_{k}^{2}(M) \\
& \tilde{S}_{k}^{2}(M)=d\left\{\left(\tilde{P}_{4, k-1}\left(\zeta_{1}, \zeta_{2}, \zeta_{3}, \theta\right) \otimes \theta\right)\left(\zeta_{1} d \zeta_{2}-\zeta_{2} d \zeta_{1}\right)\right\} \\
& \tilde{T}_{k}^{2}(M)=\underset{l+m=k}{\oplus}\left\{\begin{array}{l}
\zeta_{2}^{l} \zeta_{3}^{m} d \zeta_{2} \wedge d \zeta_{3} \\
\zeta_{1}^{l} \zeta_{3}^{m} d \zeta_{1} \wedge d \zeta_{3}
\end{array}\right. \\
& \oplus \underset{\substack{l+m+n=k \\
1 \leq m \leq k}}{\oplus}\left\{\begin{array}{l}
\zeta_{2}^{m} d \zeta_{3}^{n+1} \wedge d \zeta_{1}^{l+1}-\zeta_{3}^{n+1} d \zeta_{1}^{l+1} \wedge d \zeta_{2}^{m} \\
\zeta_{1}^{m} d \zeta_{3}^{n+1} \wedge d \zeta_{1}^{l+1}
\end{array}\right.
\end{aligned}
$$

The dimension of $Z_{k}^{2}(M)$ can then be computed as

$$
\operatorname{dim} Z_{k}^{2}(M)=\frac{1}{24}(1+k)(2+k)\left(k^{2}+15 k+48\right)
$$

We are then in a position to create all of the spaces of the graded algebra from these null spaces and the Koszul operator, ie:

$$
\begin{aligned}
& \Omega_{k_{0} k_{2}}^{1}(M)=d \Omega_{k_{0}}^{0}(M) \oplus \kappa\left(Z_{k_{2}}^{2}(M)\right) \\
& \Omega_{k_{2} k_{3}}^{2}(M)=Z_{k_{2}}^{2}(M) \oplus \kappa\left(\Omega_{k_{3}}^{3}(M)\right)
\end{aligned}
$$

In the classification of Gragial et al. [15] these bases can be considered to be type B spaces. In the following we demonstrate this case via a low order example:

2.6.1 Example: $k_{0}=1, k_{2}=0, k_{3}=0$

For the lowest order we have $\left(k_{0}=1, k_{2}=0, k_{3}=0\right)$ and we obtain the following 5 -dimensional 0 -form space:

$$
\Omega_{1}^{0}(M)=\left\{1, \zeta_{1}, \zeta_{2}, \zeta_{3}\right\} \oplus\left\{\begin{array}{l}
\zeta_{2}\left(\zeta_{1}+\zeta_{3}\right), \zeta \in T_{1} \\
\zeta_{1}\left(\zeta_{2}+\zeta_{3}\right), \zeta \in T_{2}
\end{array}\right\}
$$

We can then define a complete space of 3 -forms $\left(k_{3}=0\right)$, similarly, so we have

$$
\Omega_{0}^{3}=d \zeta_{1} \wedge d \zeta_{2} \wedge d \zeta_{3}
$$

Note that now the exterior derivative of all 3-forms vanish so this space is also the null space of 3-forms, ie. $Z_{0}^{3}=\Omega_{0}^{3}$. The null space of 1 -forms is then readily computed from $Z_{1}^{1}=d \Omega_{1}^{0}$, giving

$$
Z_{1}^{1}(M)=\left\{d \zeta_{1}, d \zeta_{2}, d \zeta_{3}\right\} \oplus\left\{\begin{array}{l}
\left(\zeta_{1}+\zeta_{3}\right) d \zeta_{2}+\zeta_{2}\left(d \zeta_{1}+d \zeta_{3}\right), \zeta \in T_{1} \\
\left(\zeta_{2}+\zeta_{3}\right) d \zeta_{1}+\zeta_{1}\left(d \zeta_{2}+d \zeta_{3}\right), \zeta \in T_{2}
\end{array}\right\}
$$

What remains is to compute the null space $Z_{0}^{2}$ of 2-forms $\left(k_{2}=0\right)$. That is

$$
Z_{0}^{2}(M)=\tilde{Z}_{0}^{2}\left(T_{3}\right) \oplus \tilde{T}_{0}^{2}(M)
$$


which gives us

$$
Z_{0}^{2}(M)=\left\{d \zeta_{2} \wedge d \zeta_{3}, d \zeta_{3} \wedge d \zeta_{1}, d \zeta_{1} \wedge d \zeta_{2}\right\} \oplus\left\{\begin{array}{c}
-d \zeta_{2} \wedge d \zeta_{3}, \zeta \in T_{1} \\
d \zeta_{3} \wedge d \zeta_{1}, \zeta \in T_{2}
\end{array}\right\}
$$

Application of the Koszul operator to (2.66) and (2.69) gives

$$
\begin{gathered}
\kappa\left(Z_{0}^{2}\right)=\left\{\zeta_{3} d \zeta_{2}-\zeta_{2} d \zeta_{3}, \zeta_{1} d \zeta_{3}-\zeta_{3} d \zeta_{1}, \zeta_{2} d \zeta_{1}-\zeta_{1} d \zeta_{2}\right\} \\
\oplus\left\{\begin{array}{l}
\zeta_{3} d \zeta_{2}-\zeta_{2} d \zeta_{3}, \zeta \in T_{1} \\
\zeta_{1} d \zeta_{3}-\zeta_{3} d \zeta_{1}, \zeta \in T_{2}
\end{array}\right\}
\end{gathered}
$$

and

$$
\kappa\left(\Omega_{0}^{3}\right)=\zeta_{1} d \zeta_{2} \wedge d \zeta_{3}+\zeta_{2} d \zeta_{3} \wedge d \zeta_{1}+\zeta_{3} d \zeta_{1} \wedge d \zeta_{2}
$$

Combining these expressions we finally have the 8-dimensional 1-form discrete function space (DFS):

$$
\begin{gathered}
\Omega_{10}^{1}=\left\{d \zeta_{1}, d \zeta_{2}, d \zeta_{3}, \zeta_{3} d \zeta_{2}-\zeta_{2} d \zeta_{3}, \zeta_{1} d \zeta_{3}-\zeta_{3} d \zeta_{1}, \zeta_{2} d \zeta_{1}-\zeta_{1} d \zeta_{2}\right\} \\
\oplus\left\{\begin{array}{l}
\left(\zeta_{1}+\zeta_{3}\right) d \zeta_{2}+\zeta_{2}\left(d \zeta_{1}+d \zeta_{3}\right), \zeta \in T_{1} \\
\left(\zeta_{2}+\zeta_{3}\right) d \zeta_{1}+\zeta_{1}\left(d \zeta_{2}+d \zeta_{3}\right), \zeta \in T_{2}
\end{array}\right\} \\
\oplus\left\{\begin{array}{l}
\zeta_{3} d \zeta_{2}-\zeta_{2} d \zeta_{3}, \zeta \in T_{1} \\
\zeta_{1} d \zeta_{3}-\zeta_{3} d \zeta_{1}, \zeta \in T_{2}
\end{array}\right\}
\end{gathered}
$$

and the 5-dimensional 2-form DFS:

$$
\begin{gathered}
\Omega_{00}^{2}=\left\{d \zeta_{2} \wedge d \zeta_{3}, d \zeta_{3} \wedge d \zeta_{1}, d \zeta_{1} \wedge d \zeta_{2}, \zeta_{1} d \zeta_{2} \wedge d \zeta_{3}+\zeta_{2} d \zeta_{3} \wedge d \zeta_{1}+\zeta_{3} d \zeta_{1} \wedge d \zeta_{2}\right\} \\
\oplus\left\{\begin{array}{c}
-d \zeta_{2} \wedge d \zeta_{3}, \zeta \in T_{1} \\
d \zeta_{3} \wedge d \zeta_{1}, \zeta \in T_{2}
\end{array}\right\}
\end{gathered}
$$

Note that the dimension of $\Omega_{10}^{1}$ is equal to the number of edges and the dimension of $\Omega_{00}^{2}$ is equal to the number of faces, as would be expected for this low order case.

\section{Construction of hierarchical conforming bases}

Clearly, the spaces constructed in Section 2.2, whilst satisfying the appropriate trivial cohomology on a single element, must be patched together appropriately across any given triangulation of the solution domain so as to match the cohomological (via duality with homology) structure of the solution domain. It is shown in [4] that a sufficient condition for this is that the trace at inter-element boundaries is continuous. We must, then, establish an appropriate basis for these spaces such that this continuity is achieved.

To this end we begin by defining appropriate degrees of freedom, a geometric decomposition onto submanifolds (Section 3.1), hierarchical test forms and hierarchical projection operators (Sections 3.2-3.5). Recursive inversion of the projection operators eventually lead to explicit form of the hierarchical basis functions. 


\subsection{Degrees of Freedom}

On one level, the degrees of freedom associated with an element $M$ are simply the coefficients multiplying the elements of the function space $\Omega^{r}(M)$ defined in section 2 . Generally speaking, however, degrees of freedom are linear functionals $L_{i}: \Omega^{r}(M) \rightarrow \mathbb{R}$ on $\Omega^{r}(M)$. (Note that in the following section we will drop any reference to polynomial order to simplify our notation.) Since the $\Omega^{r}(M)$ constitute a Hilbert space with an inner product we can use Riesz theorem to define degrees of freedom as

$$
L_{i}(\omega)=\left(\omega, \eta_{i}\right) \equiv \int_{M} \omega \wedge * \eta_{i} \text { for exactly one } \eta_{i} \in \Omega^{r}(M)
$$

Essentially, specification of the degrees of freedom becomes a process of specifying the appropriate $\eta_{i}$. We now introduce a decomposition for which this is possible. If we define the space of $r$-forms whose trace vanishes on the boundary $\partial M$ as $\Omega^{r, 0}(M)$, then we can decompose $\Omega^{r}(M)$ as follows:

$$
\Omega^{r}(M) \cong \frac{\Omega^{r}(M)}{\Omega^{r, 0}(M)} \oplus \Omega^{r, 0}(M)
$$

The quotient in equation (3.2) can be identified with the set of boundary values, and as such is isomorphic ( [26]) to the trace space $\operatorname{tr}_{\partial M}\left(\Omega^{r}(M)\right)$, hence

$$
\Omega^{r}(M) \cong \operatorname{tr}_{\partial M}\left(\Omega^{r}(M)\right) \oplus \Omega^{r, 0}(M)
$$

where $\operatorname{tr}_{\partial M}\left(\Omega^{r}(M)\right) \cong \Omega^{r}(\partial M)$.

Note that generally $\partial M$ will itself be a union of submanifolds. Hence we may apply a similar decomposition to each of these submanifolds. This process is limited since we cannot support an $r$-form on a submanifold of dimension less than $r$. Following this approach and considering $M$ as a complex $K$, so that

$$
K=\bigcup_{i=r}^{m} \bigcup_{j} \sigma_{i, j}
$$

where $\sigma_{i, j}$ is the $j$-th $i$-dimensional submanifold. Note that $m=\operatorname{dim} M, M \equiv \sigma_{m, 1}$ and $\operatorname{dim} \sigma_{i, j}=i$, so that up to isomorphism

$$
\Omega^{r}(M) \cong \Omega^{r, 0}\left(\sigma_{m, 1}\right) \oplus\left(\underset{j}{\oplus} \Omega^{r, 0}\left(\sigma_{m-1, j}\right)\right) \oplus \cdots \oplus\left(\underset{j}{\oplus} \Omega^{r, 0}\left(\sigma_{r+1, j}\right)\right) \oplus\left(\underset{j}{\oplus} \Omega^{r}\left(\sigma_{r, j}\right)\right)
$$

For example, applying this decomposition to 1-forms in 2-dimensions we have

$$
\Omega^{1}(M) \cong \Omega^{1,0}\left(\sigma_{2,1}\right) \oplus\left(\underset{j}{\oplus} \Omega^{1}\left(\sigma_{1, j}\right)\right)
$$


whereas for 1-forms in 3-dimensions we have

$$
\Omega^{1}(M) \cong \Omega^{1,0}\left(\sigma_{3,1}\right) \oplus\left(\underset{j}{\oplus} \Omega^{1,0}\left(\sigma_{2, j}\right)\right) \oplus\left(\underset{j}{\oplus} \Omega^{1}\left(\sigma_{1, j}\right)\right)
$$

In the case of 2-forms in 3-dimensions we have the following decomposition

$$
\Omega^{2}(M) \cong \Omega^{2,0}\left(\sigma_{3,1}\right) \oplus\left(\underset{j}{\oplus} \Omega^{2}\left(\sigma_{2, j}\right)\right)
$$

Given this decomposition we can define degrees of freedom as:

Definition 3.1. Degrees of Freedom

$$
\begin{gathered}
L_{\sigma_{r, j}}^{v}(\omega) \equiv \int_{\sigma_{r, j}} \omega \wedge *_{r, j} \eta_{v}^{\sigma_{r, j}}\left\{\begin{array}{c}
\eta_{v}^{\sigma_{r, j}} \in \Omega^{r}\left(\sigma_{r, j}\right), \forall j, \\
v=1, \ldots, \operatorname{dim} \Omega^{r}\left(\sigma_{r, j}\right)
\end{array}\right. \\
L_{\sigma_{i, j}}^{v}(\omega) \equiv \int_{\sigma_{i, j}} \omega \wedge *_{i, j} \eta_{v}^{\sigma_{i, j}}\left\{\begin{array}{c}
\eta_{v}^{\sigma_{i, j}} \in \Omega^{r, 0}\left(\sigma_{i, j}\right), \forall j, \\
v=1, \ldots, \operatorname{dim} \Omega^{r, 0}\left(\sigma_{i, j}\right) \\
i=r+1, \ldots, \operatorname{dim} M
\end{array}\right.
\end{gathered}
$$

where the subscript on the Hodge operator denotes a Hodge based on the induced metric over the $j$-th $i$-dimensional submanifold. The $\eta_{v}^{\sigma_{i, j}}$ are test $r$-forms on the $j$-th $i$-dimensional submanifold and span subspaces of the canonical space $\Omega^{r}(M)$. These degrees of freedom differ slightly from the moment type used conventionally in that they take the form of a scalar product and that the test forms $\eta_{v}^{\sigma_{i, j}}$ are traces of members of $\Omega^{r}(M)$. As a result, this ensures unisolvency since the test bases span the same spaces as the traces of $\Omega^{r}(M)$ and the $\Omega^{r}(M)$ themselves are linearly independent as has been shown in Section 2.2. It is important to recognize the context in which the metric (via the scalar product) is introduced. Metrical issues play no fundamental role in the construction of these function spaces. Indeed the metric, in this context, arises as nothing more than a computational tool for the enforcement of the appropriate continuity at element boundaries required to satisfy topological properties. It should be noted that the degrees of freedom $L_{i}(\omega)$ and $L_{\sigma_{r, j}}^{v}(\omega)$ are equivalent in the sense that

$$
L_{i}(\omega)=0 \forall i \Longleftrightarrow L_{\sigma_{i, j}}^{v}(\omega)=0 \forall v, i, j
$$

The specific forms of the basis functions are only defined once we have specified these test forms. Note that in order to preserve the generality of this approach, we do not a priori specify these test forms, as is usually the case, but instead construct them algorithmically from the underlying canonical function space. This allows one to construct appropriate spaces even when edges or faces have different orders of approximation or geometry, as, for instance is the case when constructing pyramidal elements. 


\subsection{Hierarchical bases}

All hierarchical spaces can be constructed by the recursive subtraction of projections of lower order spaces, as explained in [17]. The construction of hierarchical bases which span the spaces defined in Section 2 then requires the specification of a projection operator $\Pi_{k}^{r, m}: \Omega^{r} \rightarrow \Omega_{k}^{r}$ which possesses the following hierarchical property:

$$
\Pi_{k^{\prime}}^{r, m}\left(\Pi_{k}^{r, m} \omega\right)=\Pi_{k}^{r} \omega, \forall k^{\prime}>k
$$

In the following sections we explicitly define this operator in terms of appropriate hierarchical bases $\omega_{k, v}^{\sigma_{i, j}}$ and their traces on submanifolds.

\subsection{Projection}

We begin by defining a general $k^{\text {th }}$ order homogeneous projection $\tilde{\Pi}_{k}^{r, m}$ as

\section{Definition 3.2.}

$$
\tilde{\Pi}_{k}^{r, m} \omega:=\sum_{i=r j=1}^{m} \sum_{v=1}^{n_{i}} \sum_{k}^{n_{k}^{i, j}} \frac{\left(\omega, \eta_{k, v}^{\sigma_{i, j}}\right)_{\sigma_{i, j}}}{\left(\eta_{k, v}^{\sigma_{i, j}}, \eta_{k, v}^{\sigma_{i, j}}\right)_{\sigma_{i, j}}} \omega_{k, v}^{\sigma_{i, j}}
$$

where

$$
(\omega, \eta)_{\sigma_{i, j}}=\int_{\sigma_{i, j}} \omega \wedge *_{i, j} \eta
$$

and $\omega_{k, v}^{\sigma_{i, j}}$ are (as yet undetermined) global basis functions, $n_{i}$ is the number of $i$-dimensional submanifolds, $n_{k}^{i, j}=\operatorname{dim}\left(\tilde{\Omega}_{k}^{r}\left(\sigma_{i, j}\right)\right)$ if $r=i$ and $n_{k}^{i, j}=\operatorname{dim}\left(\tilde{\Omega}_{k}^{r, 0}\left(\sigma_{i, j}\right)\right)$ otherwise. Essential to this construction will be the fact that $\eta_{k, v}^{\sigma_{i, j}}=\operatorname{tr}_{\sigma_{i, j}} \omega_{k, v}^{\sigma_{i, j}}$. We then define $\Pi_{k}^{r, m} \omega:=\sum_{k^{\prime}=0}^{k} \tilde{\Pi}_{k}^{r, m} \omega$

The expression (3.13) is a valid projection (ie. satisfies (3.12)) provided

$$
\begin{gathered}
\left(\eta_{k, v^{\prime}}^{\sigma_{i, j}} \eta_{k^{\prime}, v^{\prime}}^{\sigma_{i, j}}\right)_{\sigma_{i, j}}=\delta_{k k^{\prime}} \delta_{v v^{\prime}} \\
\left(\eta_{k, v^{\prime}}^{\sigma_{i, j}} \eta_{k^{\prime}, v^{\prime}}^{\sigma_{l, n}}\right)_{\sigma_{i, j}}=0, \forall k, k^{\prime}, \forall v, v^{\prime}, l>i
\end{gathered}
$$

These are guaranteed by a construction explained later, in section 3.4. It is not necessary, but with the aim of generating well conditioned mass matrices, it can be desirable to enforce the following additional constraint on the (as yet unknown) global bases $\left\{\omega_{k, v}^{\sigma_{i, j}}\right\}$ :

$$
\left(\omega_{k, v^{\prime}}^{\sigma_{i, j}}, \eta_{k^{\prime}, v^{\prime}}^{\sigma_{l, n}}\right)_{\sigma_{l, n}}=0, \forall k^{\prime} \leq k, \forall v, v^{\prime}, l>i
$$


then we have for all $k^{\prime} \leq k$,

$$
L_{\sigma_{l, n}}^{k^{\prime}, \nu^{\prime}}\left(\omega-\tilde{\Pi}_{k}^{r, l} \omega\right)=\left(\omega, \eta_{k^{\prime}, v^{\prime}}^{\sigma_{l, n}}\right)_{\sigma_{l, n}}-\left(\omega, \eta_{k^{\prime}, v^{\prime}}^{\sigma_{l, n}}\right)_{\sigma_{l, n}}=0
$$

This projection is clearly dependent on the metric used in the definition of the Hodge star in (3.14). Importantly, we can highlight two distinct cases: Firstly, one could employ the true physical metric $g$ which depends on the particular element in question and is generally a function of the intrinsic coordinates. From this point onwards, we will denote such a projection by $\Pi_{p, h}^{r, m}$. A second option is to use a metric intrinsic to the chart coordinates. The metric in this case is flat and independent of the element and intrinsic coordinates. This makes calculation of the projection much simpler and independent of the particular element. We will usually assume that the symbol $\Pi_{h}^{r, m}$ represents the projection in this case.

\subsection{Construction of hierarchical test forms}

We begin by specifying $\Omega_{k}^{r}\left(\sigma_{r, j^{\prime}}\right)$, the space of $r$-forms on each of the $r$-dimensional submanifolds $j^{\prime}$. These are easily constructed following the procedures in section 2 . The space of $r$-forms which vanish on the boundary of $\sigma_{i, j}$ for $i>r$, are given formally by rearranging (3.3) as

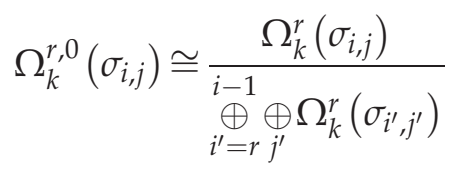

The actual computation of $\Omega_{k}^{r, 0}\left(\sigma_{i, j}\right)$ is achieved using the degrees of freedom defined in section 3.1 to construct a boundary map ; a linear transformation $\partial_{k}^{\sigma_{i, j}}: \Omega^{r}\left(\sigma_{i, j}\right) \rightarrow \Omega^{r}\left(\sigma_{i, j}\right)$, defined by:

$$
\partial_{k}^{\sigma_{i, j}} \omega=\sum_{k^{\prime}=0 i^{\prime}=r j^{\prime}=1}^{k} \sum_{v=1}^{i-1} \sum_{i_{i^{\prime}}}^{n_{k^{\prime}}^{i^{\prime} j^{\prime}}} \frac{\left(\omega, \eta_{k^{\prime}, v}^{\sigma_{i^{\prime}, j^{\prime}}}\right.}{\sigma_{\sigma_{i^{\prime}, j^{\prime}}}} \omega_{k^{\prime}, v}^{\sigma_{i^{\prime}, j^{\prime}}}
$$

The action of the boundary map is to project a function $\omega$ defined on some manifold $\sigma_{i, j}$ onto the bases associated with its boundary $\partial \sigma_{i, j}$. The kernel of $\partial_{k}^{\sigma_{i, j}}$ (ie. the null space) is precisely the space of functions which vanish on the boundary of $\sigma_{i, j}$, that is $\Omega^{r, 0}\left(\sigma_{i, j}\right)=$ $\operatorname{ker}\left(\partial_{k}^{\sigma_{i, j}}\right)$. The boundary map (3.20) acting on $\Omega_{k}^{r}\left(\sigma_{i, j}\right)$ can be written as a matrix and computing the null space of the matrix gives us the required test space $\Omega_{k}^{r, 0}\left(\sigma_{i, j}\right)$. Via this process we can construct all of the spaces of test forms required to compute the degrees of freedom in (3.9) and (3.10). This, however, is not all that we require: The desire for hierarchality is satisfied by constructing test forms which satisfy (3.15) and (3.16), so an appropriate basis for $\Omega_{k}^{r, 0}\left(\sigma_{i, j}\right)$ must be selected. To achieve this we note that the projection of the test space $\tilde{\Omega}_{k}^{r, 0}\left(\sigma_{i, j}\right)$ onto $\Omega_{k^{\prime}}^{r, 0}\left(\sigma_{i, j}\right)$ must vanish for $k^{\prime}<k$, or equivalently 
$\tilde{\Pi}_{k^{\prime}}^{r, i}\left(\Omega_{k}^{r, 0}\left(\sigma_{i, j}\right)\right)=0$ for $k^{\prime}<k$. From this we see that $\tilde{\Omega}_{k}^{r, 0}\left(\sigma_{i, j}\right)=\operatorname{ker}\left(\Pi_{k-1}^{r, i}\left(\Omega_{k}^{r, 0}\left(\sigma_{i, j}\right)\right)\right)$. All that remains is to make the $r$-forms in $\tilde{\Omega}_{k}^{r, 0}\left(\sigma_{i, j}\right)$ mutually orthogonal via a Gram-Schmidt (GS) process and we obtain hierarchical test forms $\eta_{k, v}^{\sigma_{i, j}}$ satisfying (3.15) and (3.16). We can now use these test spaces and the associated degrees of freedom to construct basis functions suited to our original purpose, as discussed in section 3.3.

\subsection{Construction of hierarchical basis forms}

We now consider the canonical shape functions $\left\{\omega_{k, \mu}\right\}$ constructed as in section 2 . These functions will generally have projections (3.13) up to order $k$. As we wish to produce hierarchical bases, we will need to construct a space $\tilde{\Omega}_{k}^{r}\left(\sigma_{m, 1}\right)$ of bases for which

$$
L_{\sigma_{l, n}}^{k^{\prime}, v^{\prime}}\left(\tilde{\Omega}_{k}^{r}\left(\sigma_{m, 1}\right)\right)=0, \forall k^{\prime}<k
$$

holds. This is easily achieved by subtracting from each $\omega_{k, \mu}$ its projection up to order $k-1$. That is we construct a homogeneous canonical space $\tilde{\Omega}_{k}^{r}\left(\sigma_{m, 1}\right)$ via,

$$
\tilde{\omega}_{k, \mu}=\omega_{k, \mu}-\Pi_{k-1}^{r, m} \omega_{k, \mu}
$$

Given this construction, we note that $\left\{\tilde{\omega}_{k, \mu}\right\}=\left\{\omega_{k, v}^{\sigma_{i, j}}\right\}$ and we can write

$$
\left\{\tilde{\omega}_{k, \mu}\right\}=\tilde{\Pi}_{k}^{r, m}\left\{\omega_{k, v}^{\sigma_{i, j}}\right\}
$$

This can be written in matrix notation as

$$
\left\{\tilde{\omega}_{k, \mu}\right\}=S\left(\left\{\tilde{\omega}_{k, \mu}\right\},\left\{\eta_{k, v}^{\sigma_{i, j}}\right\}\right)\left\{\omega_{k, v}^{\sigma_{i, j}}\right\}
$$

There is some freedom over how we may recover the $\omega_{k, v}^{\sigma_{i, j}}$. Most straightforwardly we may simply invert $S$ so that appropriate global basis functions are obtained as

$$
\left\{\omega_{k, v}^{\sigma_{i, j}}\right\}=S^{-1}\left(\left\{\tilde{\omega}_{k, \mu}\right\},\left\{\eta_{k, v}^{\sigma_{i, j}}\right\}\right)\left\{\tilde{\omega}_{k, \mu}\right\}
$$

Alternatively we may obtain a partial inverse by row reduction to form a block-upper triangular matrix. We may then obtain global bases by inversion of the individual block matrices.

The implementation of the construction of the hierarchical bases is perhaps best explained in the algorithm shown in Table 1.

Examples of 1 and 2-forms up to 2nd or 3rd order on a wide range of elements are tabulated in Section 4. Clearly, the conditioning of any resulting equations will depend on the physical problem, the geometry, element distortion $[11,15]$ and so on. However, given that this process gives rise to volume basis functions which are weakly mutually orthogonal (and in many practical cases exactly orthogonal) and that the dimension of the volume space grows faster than edge or surface spaces, then we would expect the matrices to become increasingly diagonally dominant. The conditioning of mass matrices arising from these bases is also demonstrated in Section 4. 
Table 1: Algorithm for the construction of hierarchical bases

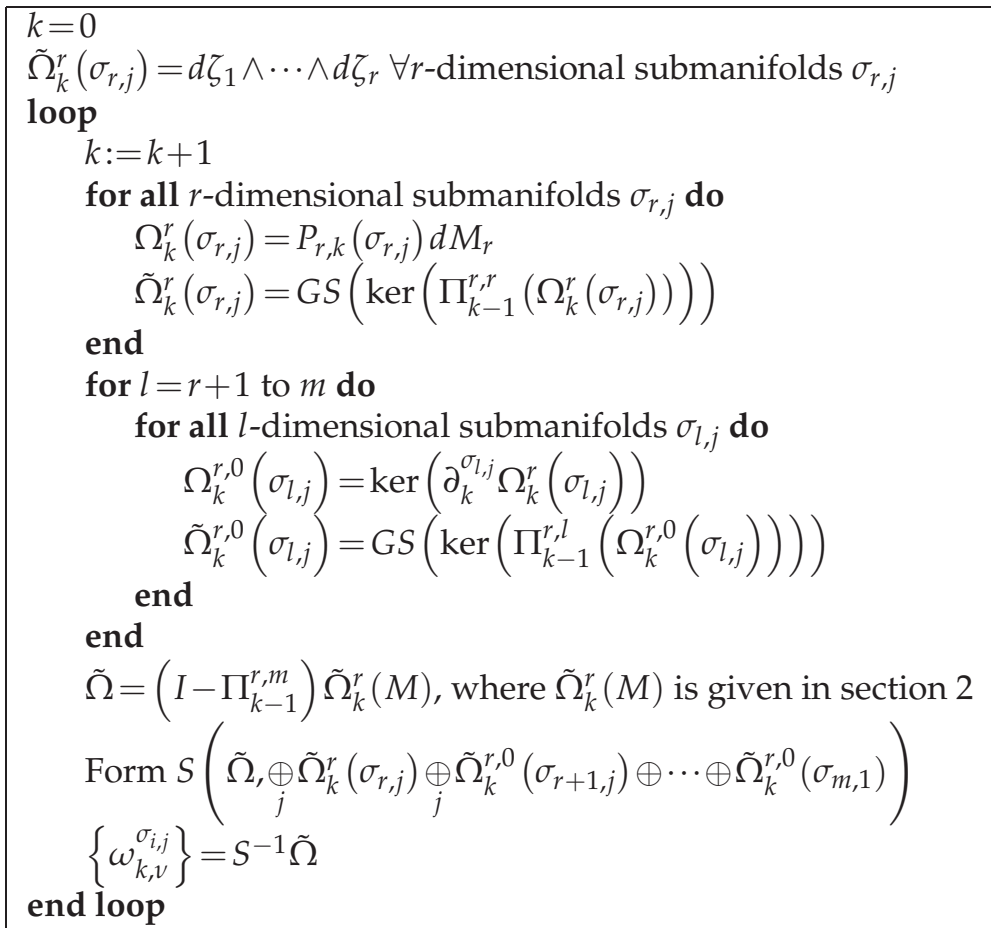

\subsection{Mathematical Properties of the Projection}

A key property often required of conformal spaces is the so-called commuting de Rham diagram (CDD) property. For the spaces constructed by [16], the CDD property is of the form

$$
d \Pi_{h}^{r} \omega=\Pi_{h}^{r+1} d \omega
$$

We will now show that the projection $\Pi_{h}^{r}$ satisfies both the CDD property and additionally a weaker adjoint form (ACDD). The ACDD property is also seen to be satisfied by the projection $\Pi_{p, h}^{r}$, but this projection only satisfies the CDD in the affine case.

Theorem 3.1. Commuting de Rham Diagram: For any smooth $\omega^{r} \in \Omega^{r}$, the projection $\Pi_{h}^{r}: \Omega^{r} \rightarrow$ $\Omega_{h}^{r}$ satisfies the commuting de Rham diagram, that is,

$$
d \Pi_{h}^{r} \omega^{r}=\Pi_{h}^{r+1} d \omega^{r}
$$

provided $d^{\dagger} \Omega_{h}^{r+1} \subset \Omega_{h}^{r}$ and $\operatorname{tr}_{\sigma_{i-1, j^{\prime}}} *_{i, j} \Omega_{h}^{r+1}\left(\sigma_{i, j}\right) \subset *_{i-1, j^{\prime}} \Omega_{h}^{r}\left(\sigma_{i-1, j^{\prime}}\right) \forall i, j$ and $j^{\prime}$.

Proof. Let us consider the projection error: $\pi^{r}=\omega^{r}-\Pi_{h}^{r} \omega^{r}$. By the definition of the canonical projection operators we see that for the $i$-dimensional subspaces $\sigma_{i, j} \in M$ which con- 
stitute subsets of bounding sub-manifolds of $M$ :

$$
\int_{\sigma_{i, j}} \pi^{r} \wedge *_{i, j} \eta^{r}=0 \forall \eta^{r} \in\left\{\begin{array}{c}
\Omega_{h, 0}^{r}\left(\sigma_{i, j}\right), r<i \leq m \\
\Omega_{h}^{r}\left(\sigma_{i, j}\right), i=r
\end{array}\right.
$$

We now consider the following expression:

$$
\begin{array}{r}
\int_{\sigma_{i, j}} d \pi^{r} \wedge *_{i, j} \eta^{r+1}=\int_{\sigma_{i, j}} \pi^{r} \wedge *_{i, j} d^{\dagger} \eta^{r+1}+\int_{\partial \sigma_{i, j}} \pi^{r} \wedge *_{i, j} \eta^{r+1} \\
\forall \eta^{r+1} \in\left\{\begin{array}{c}
\Omega_{h, 0}^{r+1}\left(\sigma_{i, j}\right), r+1<i \leq m \\
\Omega_{h}^{r+1}\left(\sigma_{i, j}\right), r+1=i
\end{array}\right.
\end{array}
$$

The first case in (3.29) is when $r+1<i \leq m$ : In this case $\eta^{r+1} \in \Omega_{h, 0}^{r+1}\left(\sigma_{i, j}\right)$ and we have that $d^{\dagger} \eta^{r+1} \in \Omega_{h}^{r}\left(\sigma_{i, j}\right)$. Hence in this case the first term on the RHS of (3.29) vanishes. The second integral also vanishes from the second provision in the theorem. The second case is when $r+1=i$ and $\eta^{r+1} \in \Omega_{h}^{r+1}\left(\sigma_{i, j}\right)$, so $d^{\dagger} \eta^{r+1} \in \Omega_{h}^{r}\left(\sigma_{i, j}\right)$ and again the first integral in (3.29) vanishes according to (3.28). The second integral also vanishes due to the second provision in the theorem. which completes the proof.

A similar argument can be made for the physical projection $\Pi_{p, h}^{r}$, provided the metrical coefficients are constant, ie. an affine case. Note - it is the conjecture here that the $\mathrm{CDD}$ is a sufficient, not necessary, condition for the convergence of numerical schemes. In fact it is easy to show that satisfaction of the CDD implies a trivial local cohomology, as is shown in [4].

Theorem 3.2. Adjoint Commuting de Rham Diagram: For any smooth $\omega^{r} \in \Omega^{r}$, the projection $\Pi_{h}^{r}: \Omega^{r} \rightarrow \Omega_{h}^{r}$ satisfies the adjoint commuting de Rham diagram, that is,

$$
\Pi_{h}^{r-1} d^{\dagger} \Pi_{h}^{r} \omega=\Pi_{h}^{r-1} d^{\dagger} \omega
$$

provided $\Omega_{h}^{r}$ has a trivial local cohomology. The same statement holds for $\Pi_{p, h}^{r}$.

Proof. Again we consider the projection error: $\pi^{r}=\omega^{r}-\Pi_{h}^{r} \omega^{r}$. By the definition of the canonical interpolation operators we see that for all of the $i$-dimensional subspaces $\sigma_{i, j} \in$ $M$ which constitute subsets of bounding sub-manifolds of $M$ :

$$
\int_{\sigma_{i, j}} \pi^{r} \wedge *_{i, j} \eta^{r}=0 \forall \eta^{r} \in\left\{\begin{array}{c}
\Omega_{h, 0}^{r}\left(\sigma_{i, j}\right), r<i \leq m \\
\Omega_{h}^{r}\left(\sigma_{i, j}\right), i=r
\end{array}\right.
$$

We now consider the following expression:

$$
\begin{array}{r}
\int_{\sigma_{i, j}} d^{\dagger} \pi^{r} \wedge *_{i, j} \eta^{r-1}=\int_{\sigma_{i, j}} \pi^{r} \wedge *_{i, j} d \eta^{r-1}-\int_{\partial \sigma_{i, j}} *_{i, j} \pi^{r} \wedge \eta^{r-1} \\
\forall \eta^{r-1} \in\left\{\begin{array}{c}
\Omega_{h, 0}^{r-1}\left(\sigma_{i, j}\right), r-1<i \leq m \\
\Omega_{h}^{r-1}\left(\sigma_{i, j}\right), r-1=i
\end{array}\right.
\end{array}
$$


The first case in (3.32) is when $r-1<i \leq m$ : In this case $\eta^{r-1} \in \Omega_{h, 0}^{r-1}\left(\sigma_{i, j}\right)$, so $d \eta^{r-1} \in \Omega_{h}^{r}\left(\sigma_{i, j}\right)$. We also have that $t r_{\partial \sigma_{i, j}} d \eta^{r-1}=d\left(t_{\partial \sigma_{i, j}} \eta^{r-1}\right)=0$ so $d \eta^{r-1} \in \Omega_{h, 0}^{r}\left(\sigma_{i, j}\right)$. The first integral in on the RHS of (3.32) then vanishes by (3.31). Moreover, since $t r_{\partial \sigma_{i, j}} \eta^{r-1}=0$ then the second integral also vanishes. The second case is when $r-1=i$ and $\eta^{r-1} \in \Omega_{h}^{r-1}\left(\sigma_{i, j}\right)$, so $d \eta^{r-1} \in \Omega_{h}^{r}\left(\sigma_{i, j}\right)$ and again the first integral in (3.32) vanishes according to (3.31). The second integral also vanishes since $*_{i, j} \pi^{r} \wedge \eta^{r-1}=*_{i, j} \pi^{i+1} \wedge \eta^{i}=0$ due to the fact that by definition there are no $i+1$ forms in $i$-dimensions. We then have that

$$
\int_{\sigma_{i, j}} d^{\dagger} \pi^{r} \wedge *_{i, j} \eta^{r-1}=0 \forall \eta^{r-1} \in\left\{\begin{array}{c}
\Omega_{h, 0}^{r-1}\left(\sigma_{i, j}\right), r-1<i \leq m \\
\Omega_{h}^{r-1}\left(\sigma_{i, j}\right), r-1=i
\end{array}\right.
$$

From which it follows that

$$
\Pi_{h}^{r-1} d^{\dagger} \Pi_{h}^{r} \omega=\Pi_{h}^{r-1} d^{\dagger} \omega
$$

as required. An identical argument holds for the projection based on the physical metric $\Pi_{p, h}^{r}$.

Note that this is a weaker statement than the conventional CDD property. If we restrict ourselves to affine elements we can show that (3.30) holds in the strict sense.

\section{Examples}

We now demonstrate the results of the procedures outlined earlier for specific cases. Bases of 1 and 2-forms on tetrahedra, hexahedra, prisms and pyramids are tabulated. To assess the performance of these bases, following Xin et al. [29] we compute the condition number of the mass, quasi-stiffness and composite matrices on a parent element. The mass (M), stiffness $(\mathrm{S})$ and composite $(\mathrm{C})$ matrices are defined as

$$
M=\left(\omega_{k, v^{\prime}}^{\sigma_{i, j}} \omega_{k^{\prime}, v^{\prime}}^{\sigma_{l, n}}\right), S=\left(d \omega_{k, v^{\prime}}^{\sigma_{i, j}} d \omega_{k^{\prime}, v^{\prime}}^{\sigma_{l, n}}\right), C=\mu M+S
$$

\subsection{Tetrahedra}

For brevity we will employ a barycentric notation, commonly employed on simplices. Bases for other edges/faces can be obtained by proper rotations via an appropriate pullback of the map

$$
\mathbf{r}_{\{i j k l\}}=\zeta_{1} \mathbf{r}_{i}+\zeta_{2} \mathbf{r}_{j}+\zeta_{3} \mathbf{r}_{k}+\left(1-\zeta_{1}-\zeta_{2}-\zeta_{3}\right) \mathbf{r}_{l}
$$

Note that proper rotations correspond to even permutations of $\{i j k l\}$. Thus, given a face function $\omega^{\{i j k\}}$ we can construct the corresponding base for $\omega^{\{j k l\}}$ via

$$
\omega^{\{j k l\}}=\mathbf{r}_{\{j k l i\}}^{*} \omega^{\{i j k\}}
$$


Similarly, given an edge function $\omega^{\{i j\}}$ we can construct the corresponding base for $\omega^{\{j k\}}$ via

$$
\omega^{\{j k\}}=\mathbf{r}_{\{j k l i\}}^{*} \omega^{\{i j\}}
$$

Type B 1- and 2-form bases on tetrahedra are given in Tables 2 and 3 respectively, up to order $k=3$. Corresponding Type $C$ bases for both 1 and 2-forms are given in Table 4 . The condition numbers of the mass, quasi stiffness and composite matrices for a single element are plotted in Figures 3 and 4 for 1 and 2-forms respectively. In Figure 3(a) we compare the growth of the mass matrix condition number for our type B 1-forms with those of Abdul-Rahman ( $\mathrm{Ab})$ [1]. In Figure 3(b) we compare the growth of mass matrix condition number for our type C 1-forms with Ingelstrom (In) [21,22]. It is clear that for the mass matrix, the conditioning of our bases is comparable to Ingelstrom (C-type), although not as good as Abdul-Rahman (B-type). The growth in the condition number appears to be exponential for the 1-forms and possibly sub-exponential for 2-forms. The composite matrix condition number reduces as the coupling parameter $\mu$ increases, as is the case in [29] for type A bases. Broadly similar behaviour for both B and C-type 2-form bases is observed in Figure 4.

\subsection{Hexahedra}

The construction of the canonical spaces on hexahedra is very simple since the construction of the discrete function space involves the specification of the discrete function space on simple one-dimensional manifolds for 1-forms and two dimensional manifolds for 2forms as discussed in section 2. The construction of the hierarchical bases then follows that outlined in section 3. For brevity, only the functions associated with edge 7 (for 1forms) and face 1 are given, where the submanifold numbering is indicated in Figure 2. Other functions can be readily obtained via a rotation pullback. The condition numbers of resulting mass, quasi-stiffness and composite matrices are shown in Figure 5. Both 1 and 2-forms exhibit exponential growth although there is evidence that 1-forms may show sub-exponential behaviour.

\subsection{Prisms}

This prism element has canonical functions given in (2.72) with manifold numbering shown in Figure 2. Basis forms are tabulated for the type B case in Table 6 and those for the type $\mathrm{C}$ case in Table 7. For brevity, only the functions associated with edges 1 and 8 (for 1-forms), and faces 1 and 3 are given. Again, bases for other edges and faces can be readily obtained via a rotation pullback. The dependence of condition number on element order of the resulting matrices is shown in Figures 6 (1-forms) and 7 (2-forms). Type B and C 1-forms exhibit exponential and sub-exponential behaviour respectively. The corresponding 2-forms (both B and $C$ types) exhibit exponential growth. It is worth also noting that the condition numbers for the composite matrices for prisms rise more 
rapidly with element order than either tetrahedra or hexahedra, implying relatively poor conditioning.

\subsection{Pyramids}

This pyramid element has canonical functions given in (2.64) with manifold numbering shown in Figure 2. The functions associated with edges 2 and 7 (for 1-forms), and faces 1 and 3 are given in Tables 8 and 9 for 1 and 2-forms respectively. The condition numbers of the resulting matrices are shown in figure 8 . In both cases we see sub-exponential growth with element order. As is the case for prisms, the composite matrices for pyramids rise more rapidly with element order than either tetrahedra or hexahedra, again implying comparatively poor conditioning. In practice, however, typical meshes would contain very few such elements (at the interface between tetrahedra and hexahedra, for instance) and it is possible that the poor conditioning of prisms and pyramids would have limited overall effect.

\section{Conclusion}

A procedure for the systematic construction of hierarchical conforming bases on a range of element types is demonstrated based on an ab initio preservation of the underlying cohomology. This process supports not only the most common simplicial element types, but is generalised to hexahedra, prisms and importantly pyramids. This generalisation is effected by recourse to basic ideas from algebraic topology (differential forms, homology, cohomology, etc) and as such extends the fundamental theoretical framework established by the work of Hiptmair [16-18] and Arnold et al. [4].

These bases can be classify as type B or C according to Graglia et al [15] for tetrahedra and prisms, depending on the choice of polynomial space parameters $k_{0}, k_{2}$ and $k_{3}$ and type $B$ for hexahedra and pyramids. The resulting bases are tabulated for a range of orders. The condition number for mass, quasi-stiffness and composite matrices is computed and where appropriate, compared with alternatives. It is seen that the condition number grows exponentially or better in all cases. Furthermore, tetrahedral and hexahedra exhibit consistently better conditioning than prisms or pyramids.

\section{References}

[1] R. Abdul-Rahman and M. Kasper. Orthogonal hierarchical Nédélec elements. IEEE Trans. Magn. (USA), 44(6):1210 - 13, 2008.

[2] L.S. Andersen and J.L. Volakis. Hierarchical tangential vector finite elements for tetrahedra. IEEE Microw. Guid. Wave Lett. (USA), 8(3):127 - 9, 1998.

[3] L.S. Andersen and J.L. Volakis. Development and application of a novel class of hierarchical tangential vector finite elements for electromagnetics. IEEE Trans. Antennas Propag. (USA), 47(1):112 - 20, 1999. 
[4] D.N. Arnold, R.S. Falk, and R. Winther. Finite element exterior calculus, homological techniques, and applications. Acta Numer. (UK), 15:1 - 155, 2006.

[5] M. J. Bluck, A. Hatzipetros, and S. P. Walker. Applications of differential forms to boundary integral equations. IEEE Trans. Antennas Propag. (USA), 54(6):1781-96, 2006.

[6] M. J. Bluck and S. P. Walker. Polynomial basis functions on pyramidal elements. Communications in Numerical Methods in Engineering, 24(12):1827-1837, 2008.

[7] A. Bossavit. Whitney forms: a class of finite elements for three-dimensional computations in electromagnetism. IEE Proc. A, Phys. Sci. Meas. Instrum. Manage. Educ. Rev. (UK), 135(8):493 $-500,1988$.

[8] A. Bossavit. Simplicial finite elements for scattering problems in electromagnetism. Comput. Methods Appl. Mech. Eng. (Netherlands), 76(3):299 - 316, 1989.

[9] A. Bossavit. Generating Whitney forms of polynomial degree one and higher. IEEE Transactions on Magnetics, 38(2, pt.1), 2002.

[10] J.W. Eastwood and J.G. Morgan. Higher-order basis functions for mom calculations. IET Sci. Meas. Technol. (UK), 2(6):379-86, 2008/11/.

[11] R. S. Falk, P. Gatto, and P. Monk. Hexahedral h(div) and h(curl) finite elements. ESAIM: Mathematical Modelling and Numerical Analysis, 45(1):115 - 143, 2011.

[12] V. Gradinaru and R. Hiptmair. Whitney elements on pyramids. Electron. Trans. Numer. Anal., 8:154-168, 1999.

[13] R. D. Graglia and I. L. Gheorma. Higher order interpolatory vector bases on pyramidal elements. IEEE Trans. Antennas Propag. (USA), 47(5):775, 1999.

[14] R. D. Graglia, D. R. Wilton, and A. F. Peterson. Higher order interpolatory vector bases for computational electromagnetics. IEEE Trans. Antennas Propag. (USA), 45(3):329, 1997.

[15] R.D. Graglia, A.F. Peterson, and F.P. Andriulli. Curl-conforming hierarchical vector bases for triangles and tetrahedra. IEEE Trans. Antennas Propag. (USA), 59(3):950-9, March 2011.

[16] R. Hiptmair. Canonical construction of finite elements. Mathematics of Computation, 68(228):1325, 1999.

[17] R. Hiptmair. Higher order Whitney forms. Journal of Electromagnetic Waves and Applications, 15(3):341, 2001.

[18] R. Hiptmair. Finite elements in computational electromagnetism. Acta Numerica, 11:237, 2002.

[19] M.M. Ilic and B.M. Notaros. Higher order hierarchical curved hexahedral vector finite elements for electromagnetic modeling. IEEE Trans. Microw. Theory Tech. (USA), 51(3):1026-33, 2003.

[20] M.M. Ilic and B.M. Notaros. Higher order large-domain hierarchical FEM technique for electromagnetic modeling using Legendre basis functions on generalized hexahedra. Electromagnetics (UK), 26(7):517 - 29, 2006.

[21] P. Ingelstrom. A new set of $\mathrm{h}$ (curl)-conforming hierarchical basis functions for tetrahedral meshes. IEEE Trans. Microw. Theory Tech. (USA), 54(1):106 - 14, 2006.

[22] P. Ingelstrom, V. Hill, and R. Dyczij-Edlinger. Comparison of hierarchical basis functions for efficient multilevel solvers. IET Sci. Meas. Technol. (UK), 1(1):48 - 52, 2007.

[23] M. Nakahara. Geometry, topology and physics. Second edition. IOP Publishing, 2003.

[24] J. C. Nédélec. Mixed finite elements in $\mathbb{R}^{3}$. Numerische Mathematik, 35(3):315, 1980.

[25] J. Schöberl and S. Zaglmayr. High order Nédélec elements with local complete sequence property. COMPEL, 24(2):374-384, 2005.

[26] R.E. Showalter. Hilbert Space Methods for Partial Differential Equations. Pitman Publishing, London, 1977. 
[27] J.P. Webb. Hierarchal vector basis functions of arbitrary order for triangular and tetrahedral finite elements. IEEE Trans. Antennas Propag. (USA), 47(8):1244 - 53, 1999.

[28] J. Xin and W. Cai. A well-conditioned hierarchical basis for triangular H(curl)-conforming elements. Commun. Comput. Phys., 9(3):780-806, 2011.

[29] J. Xin, N. Guo, and W. Cai. On the construction of well-conditioned hierarchical bases for tetrahedral H(curl)-conforming Nédélec elements. Journal of Computational Mathematics, 29(5):526-542, 2011. 

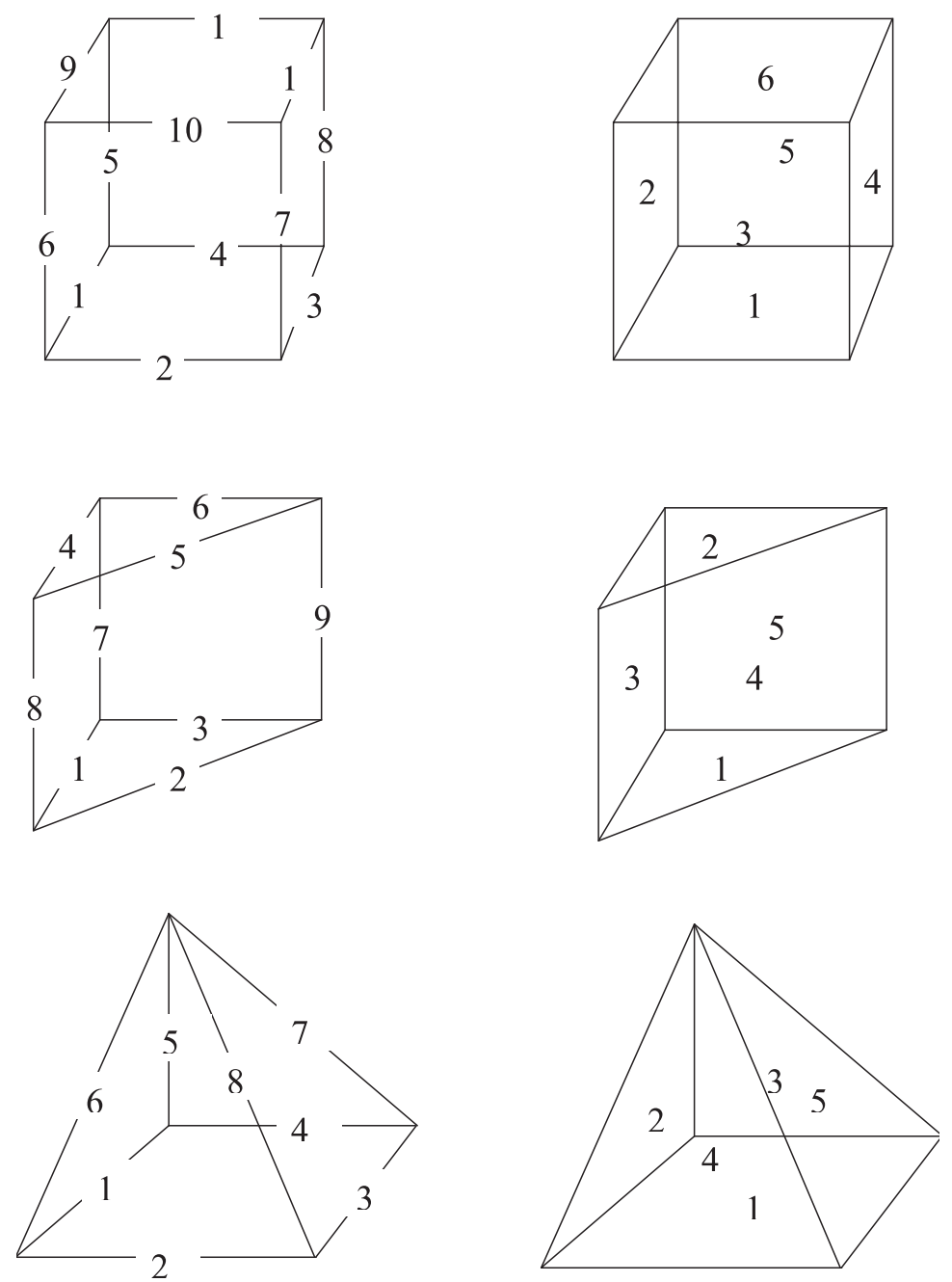

(a) $\sigma_{1, i}$

(b) $\sigma_{2, j}$

Figure 2: Submanifold numbering 
(a)

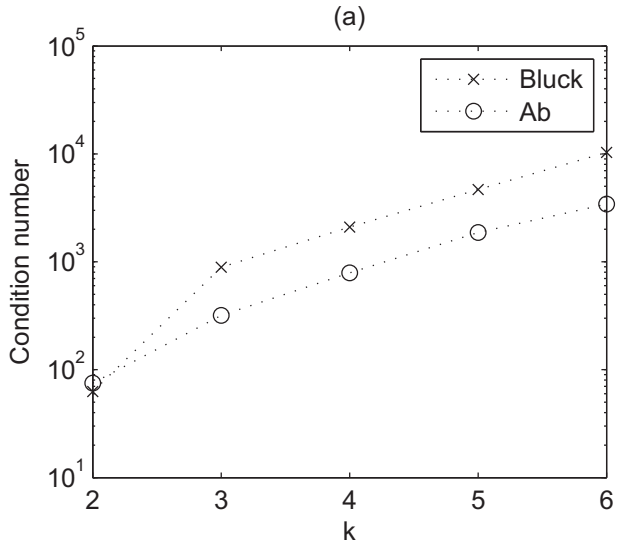

(c)

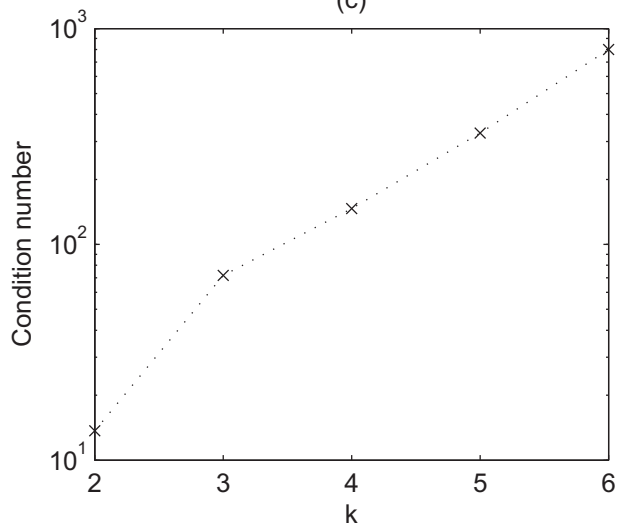

(e)

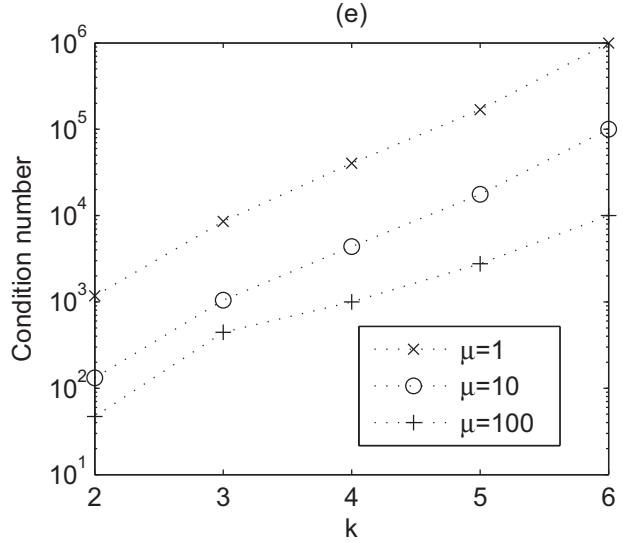

(b)

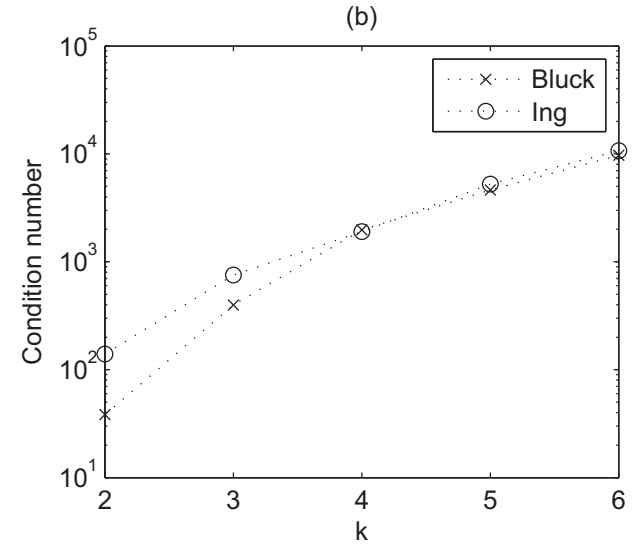

(d)
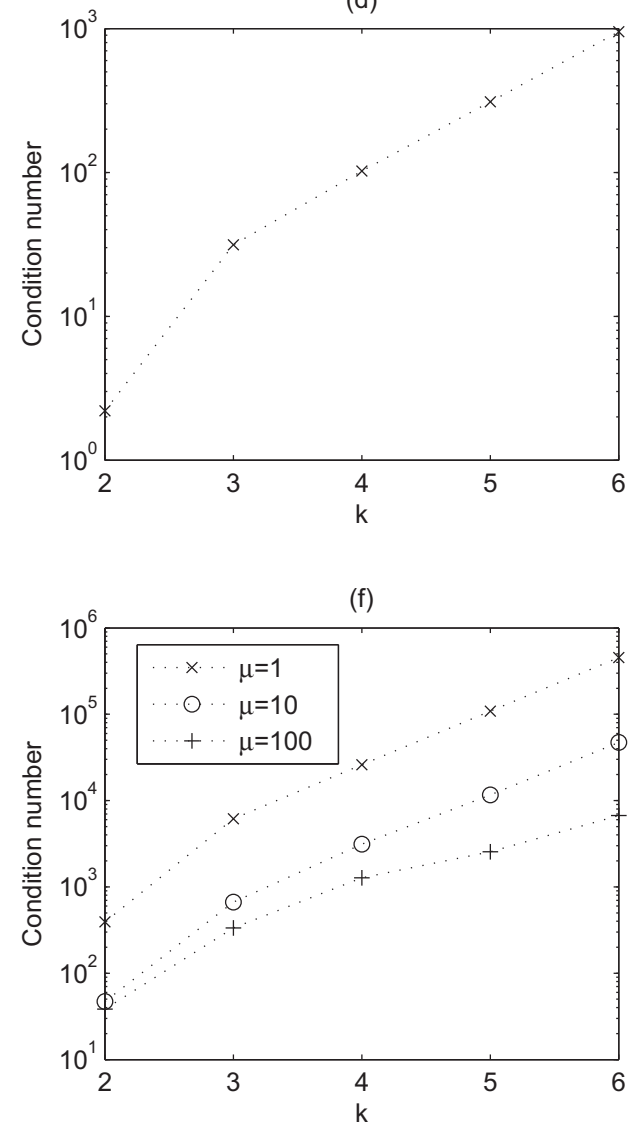

Figure 3: Condition number vs order for single element mass matrices (a), (b), quasi-stiffness matrices (c), (d) and composite matrices (e), (f) for B and C-type 1-form bases on tetrahedra respectively. 
(a)

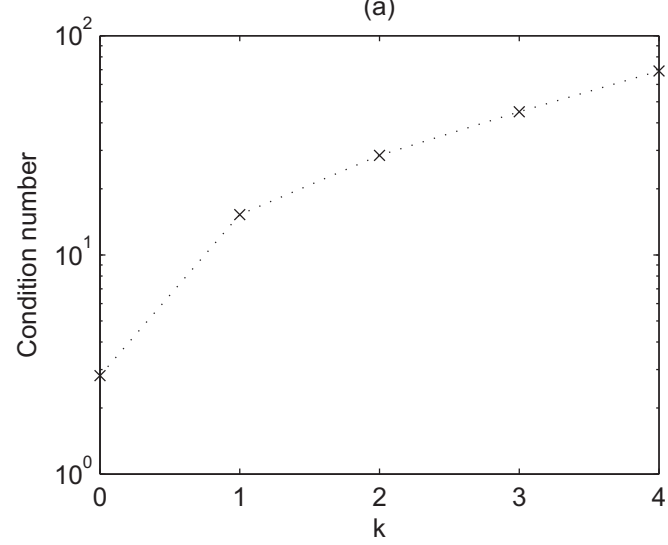

(c)

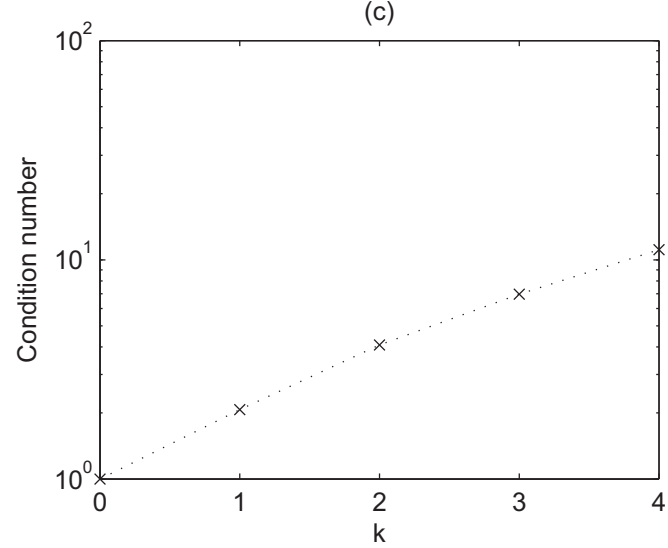

(e)

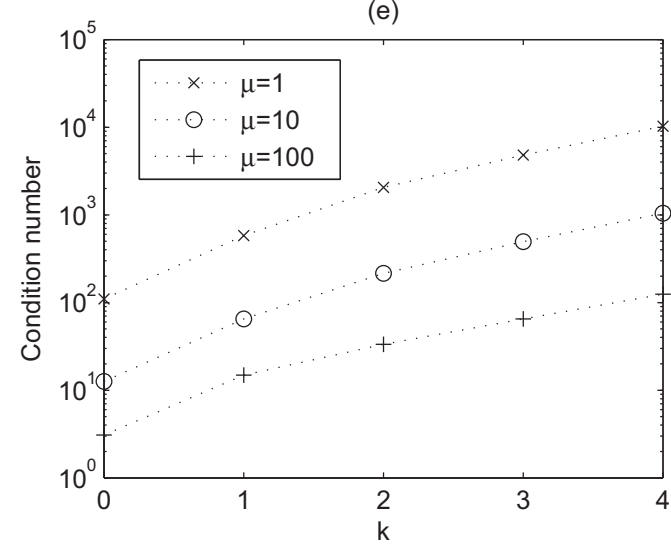

(b)

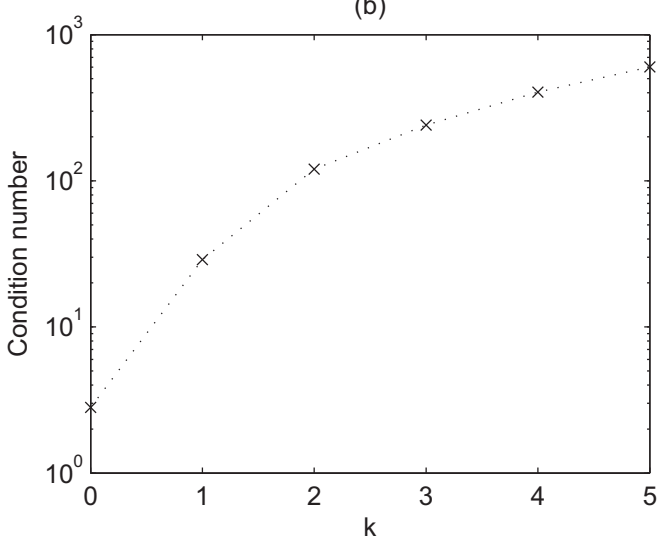

(d)

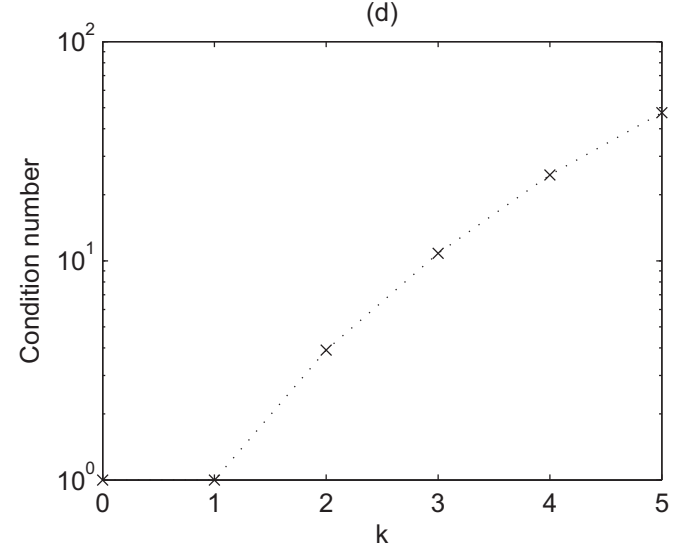

(f)

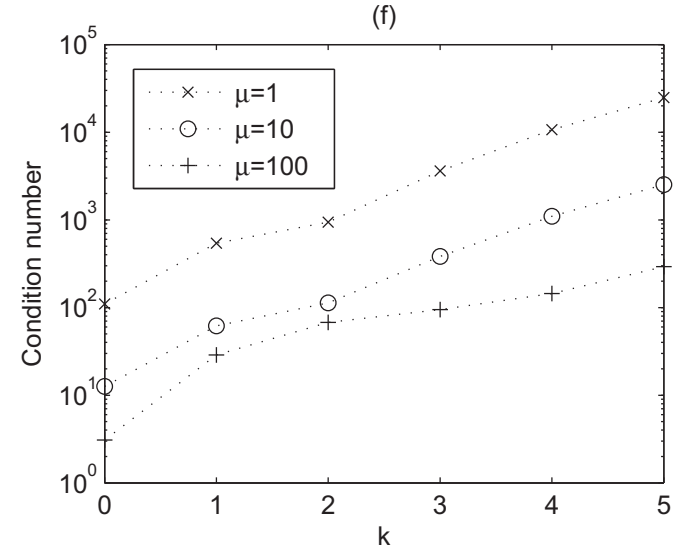

Figure 4: Condition number vs order for single element mass matrices (a), (b), quasi-stiffness matrices (c), (d) and composite matrices (e), (f) for B and C-type 2-form bases on tetrahedra respectively. 
(a)

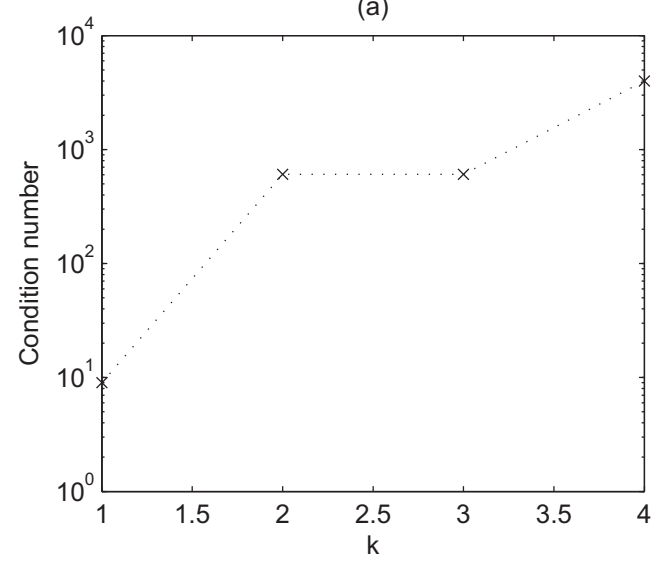

(b)

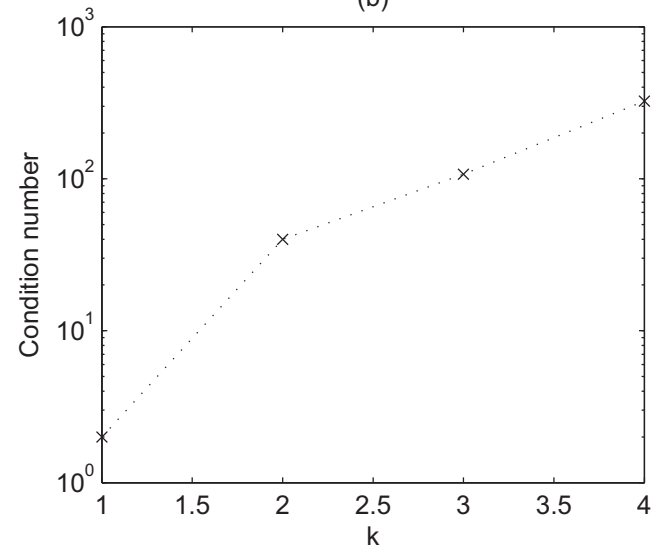

(c)

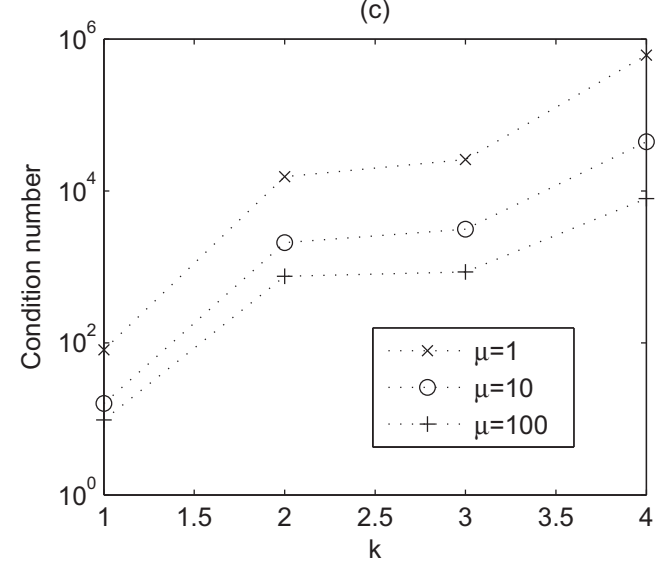

(d)

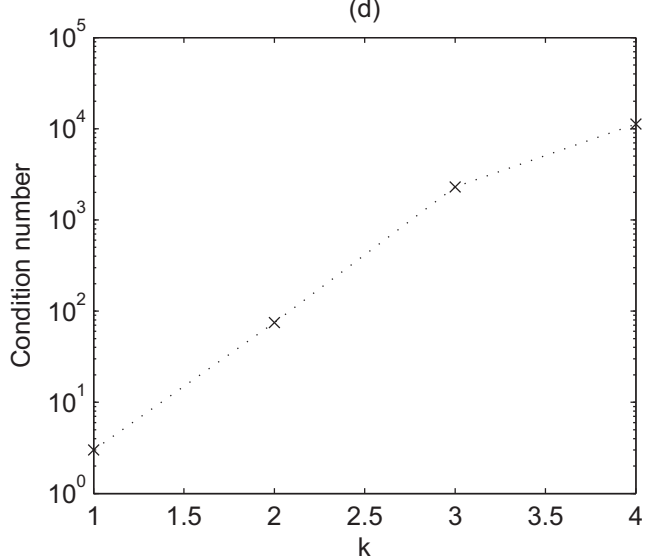

(e)

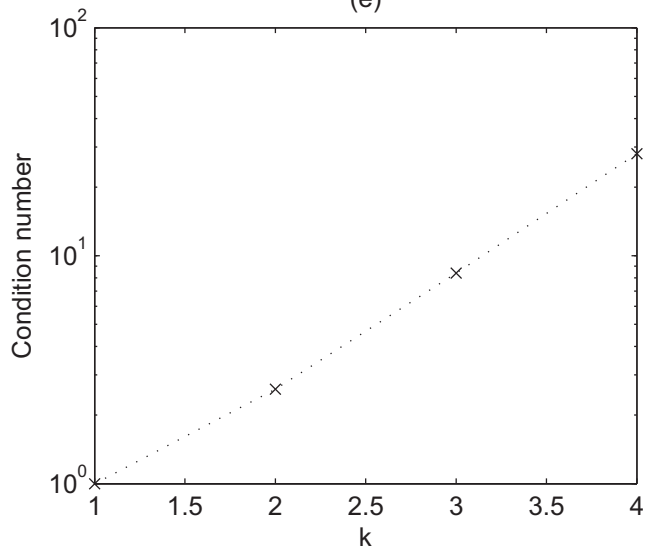

(f)

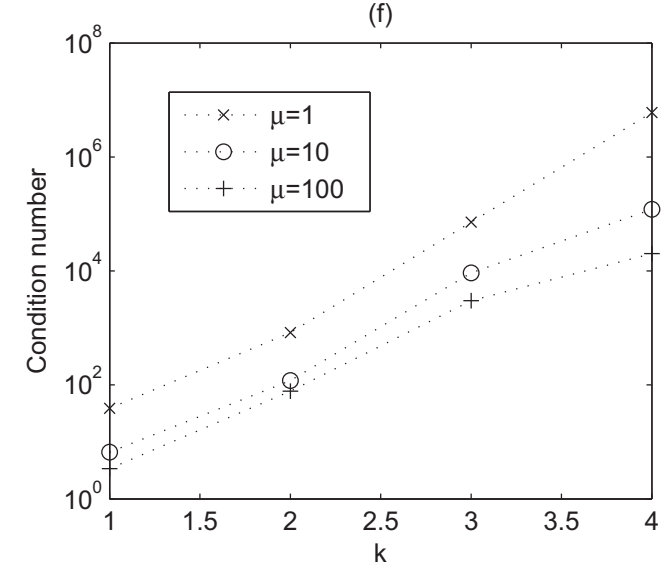

Figure 5: Condition number vs order for single element mass matrices, quasi-stiffness matrices and composite matrices for 1-form bases ((a), (b), (c )) and 2-form bases ((d),(e),(f)) on hexahedra. 
(a)

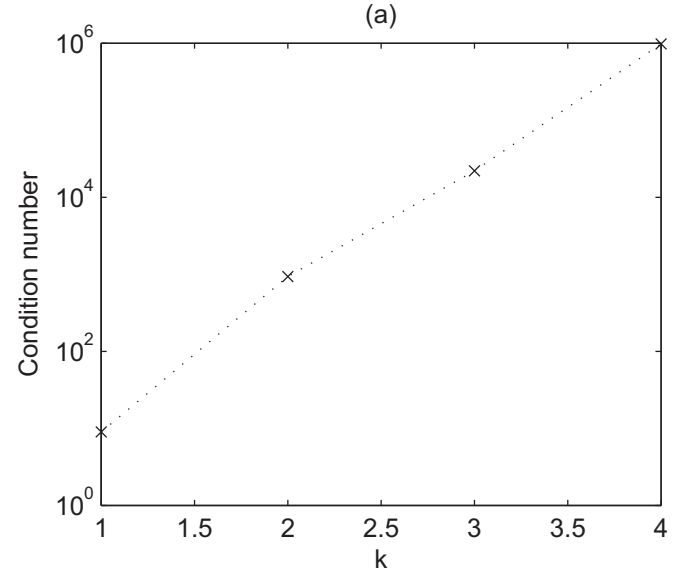

(c)

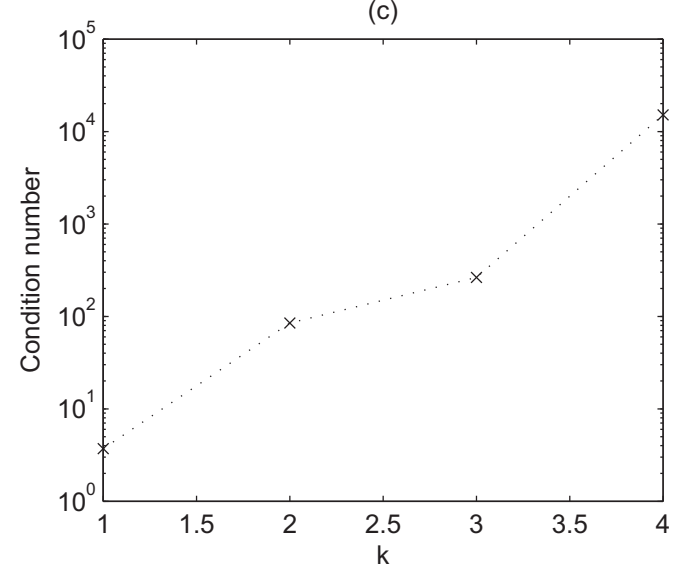

(e)

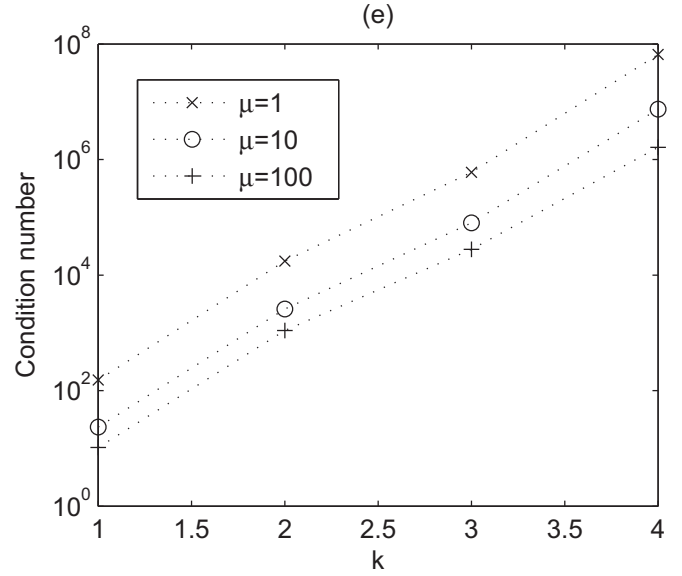

(b)

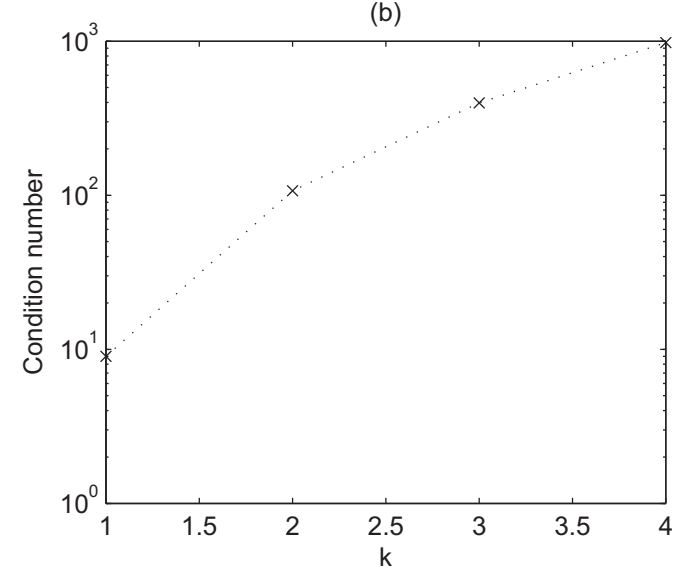

(d)

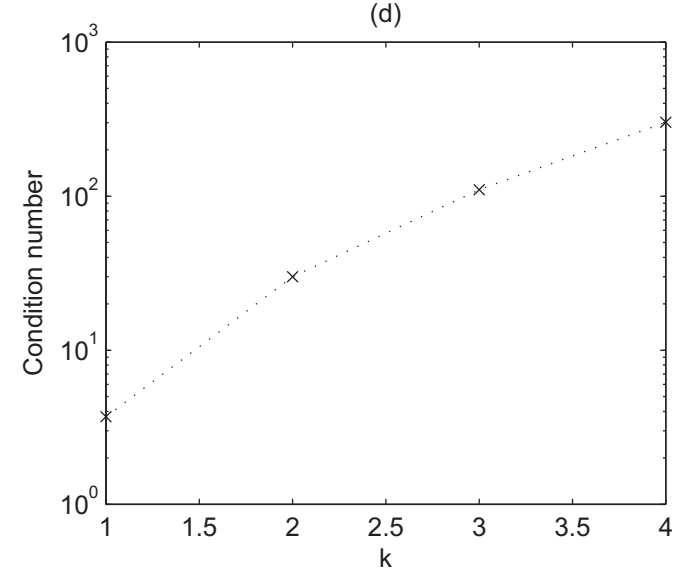

(f)

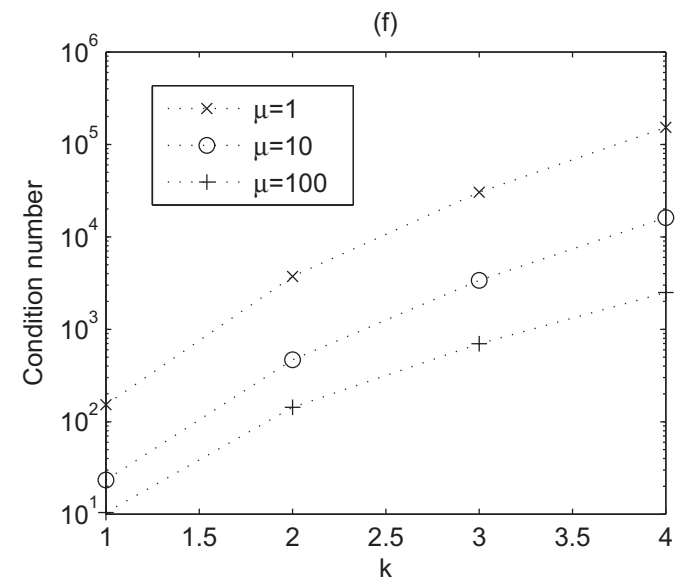

Figure 6: Condition number vs order for single element mass matrices (a), (b), quasi-stiffness matrices (c), (d) and composite matrices (e), (f) for B and C-type 1-form bases on prisms. 
(a)

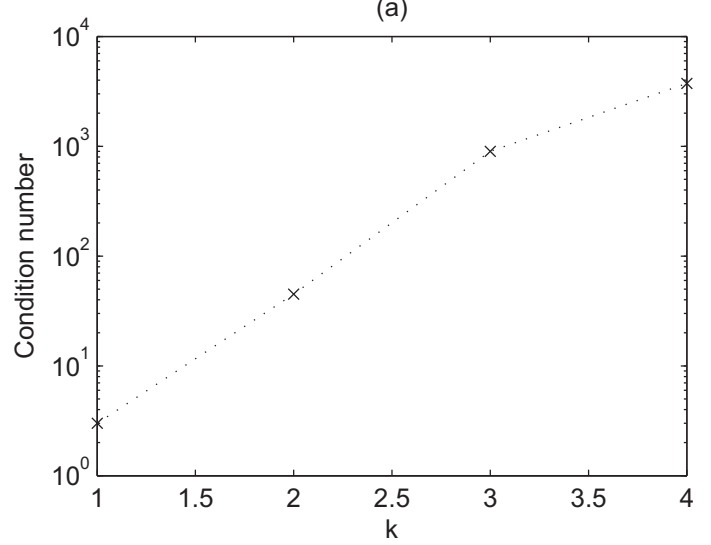

(c)

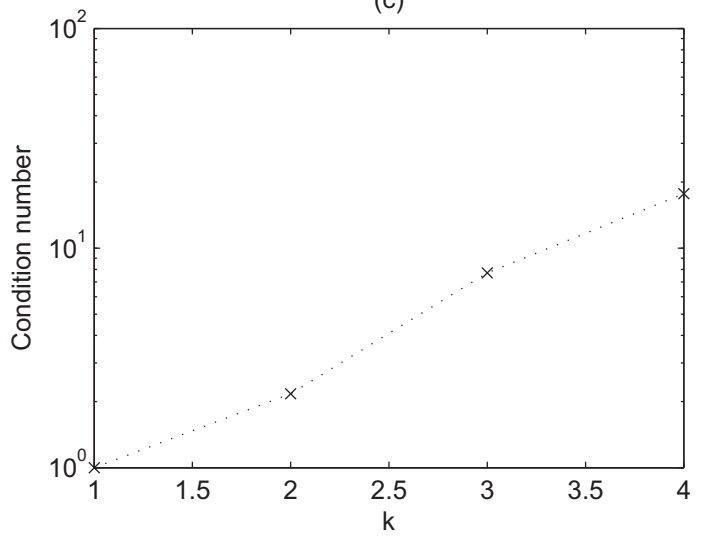

(e)

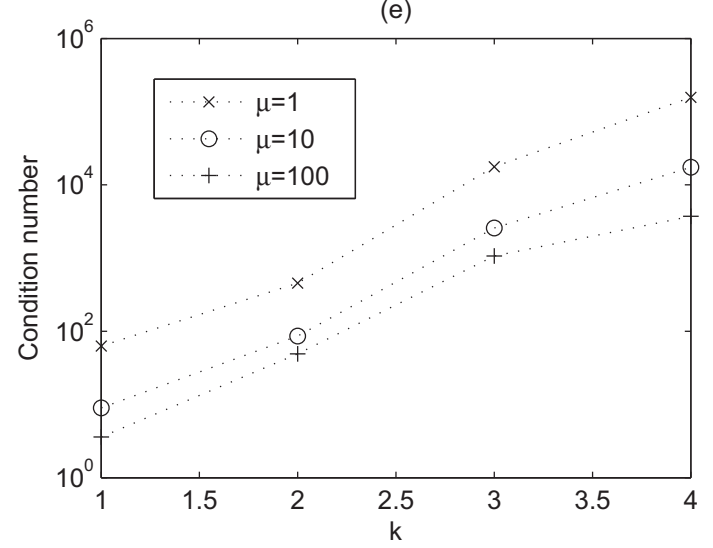

(b)

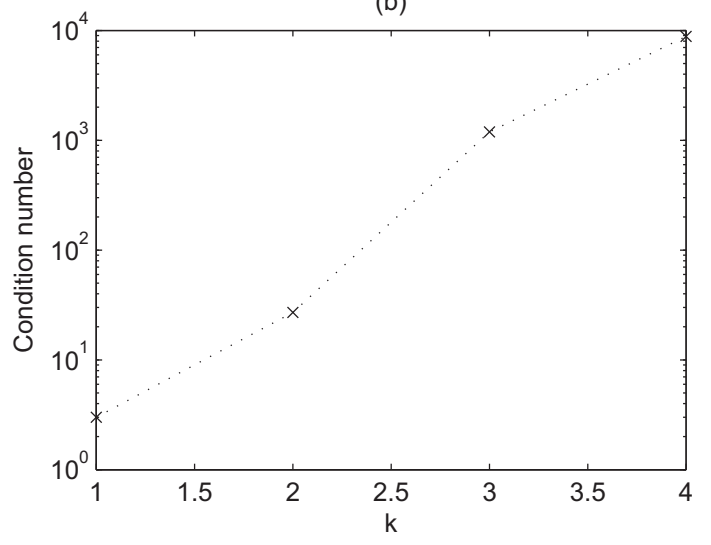

(d)

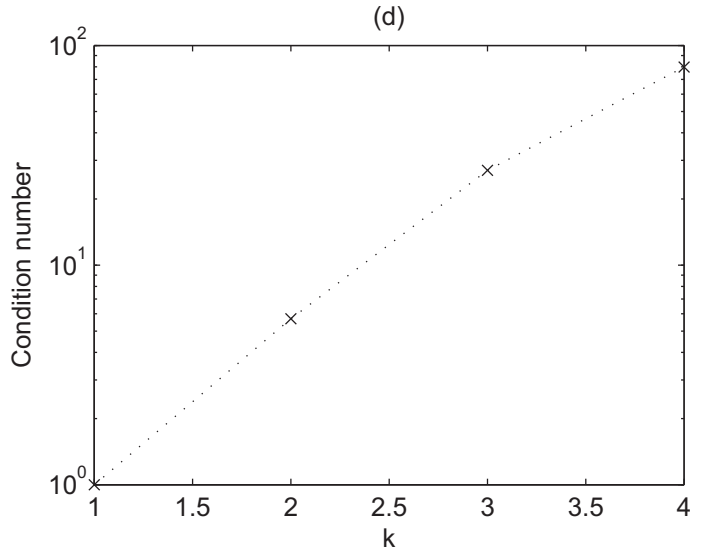

(f)

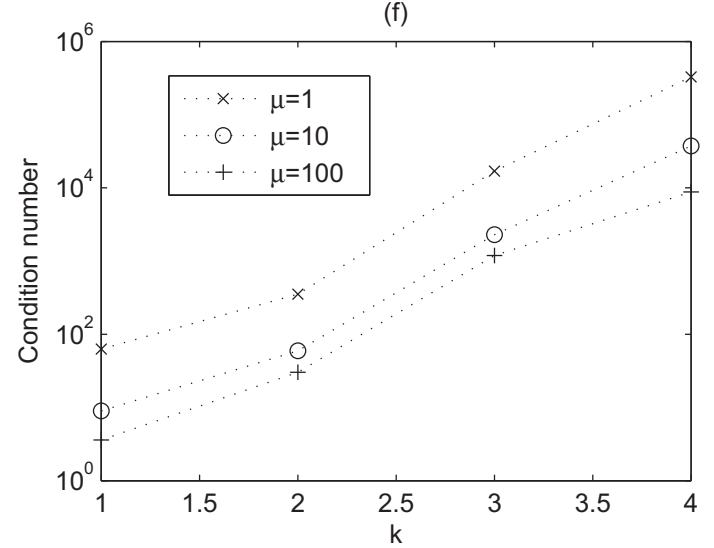

Figure 7: Condition number vs order for single element mass matrices (a), (b), quasi-stiffness matrices (c), (d) and composite matrices (e), (f) for B and C-type 2-form bases on prisms. 
(a)

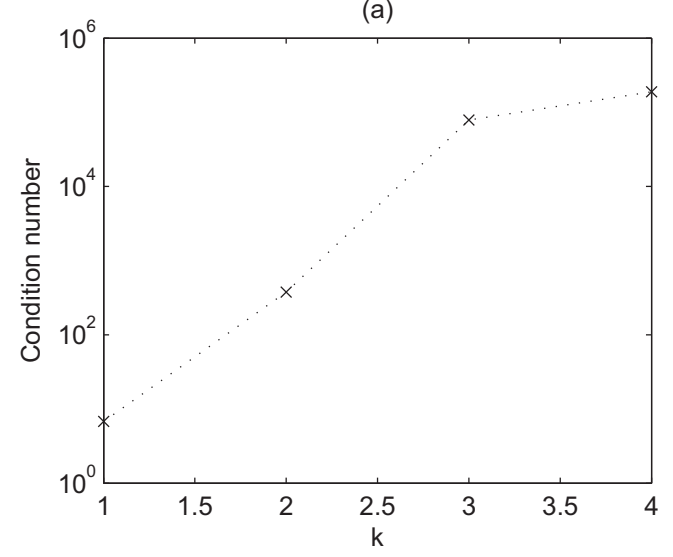

(b)

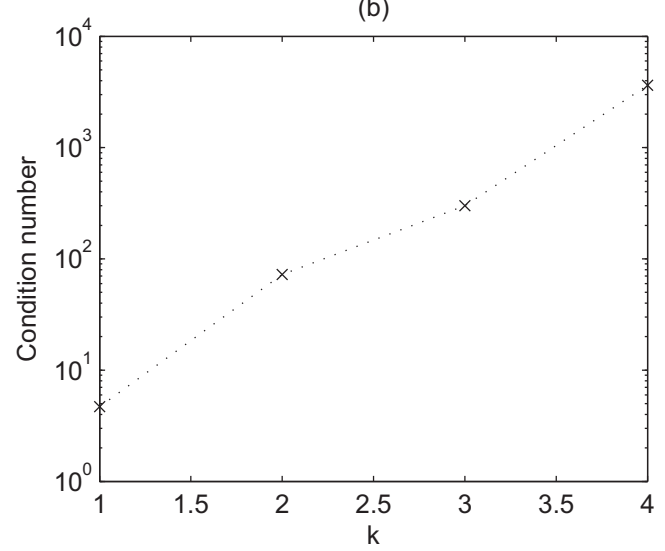

(c)

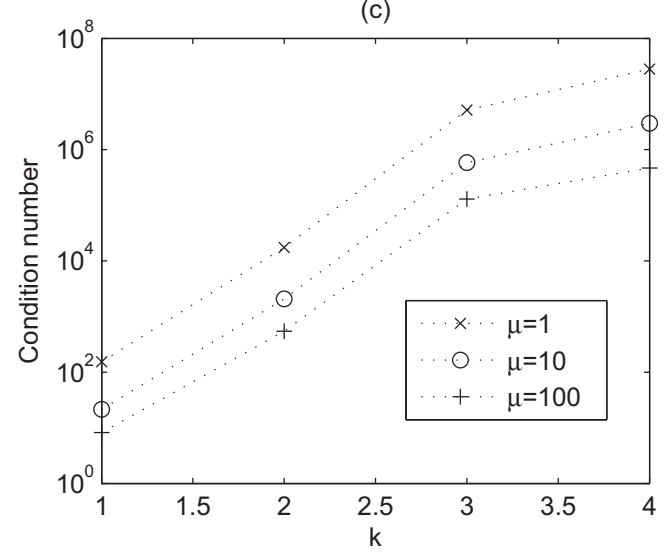

(d)

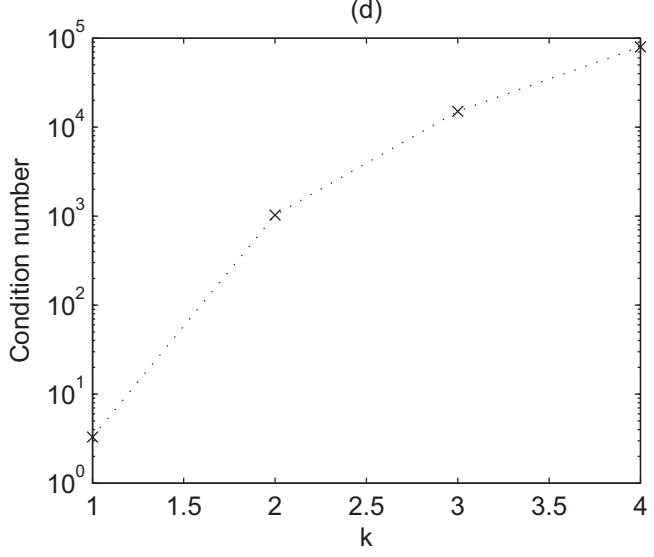

(e)
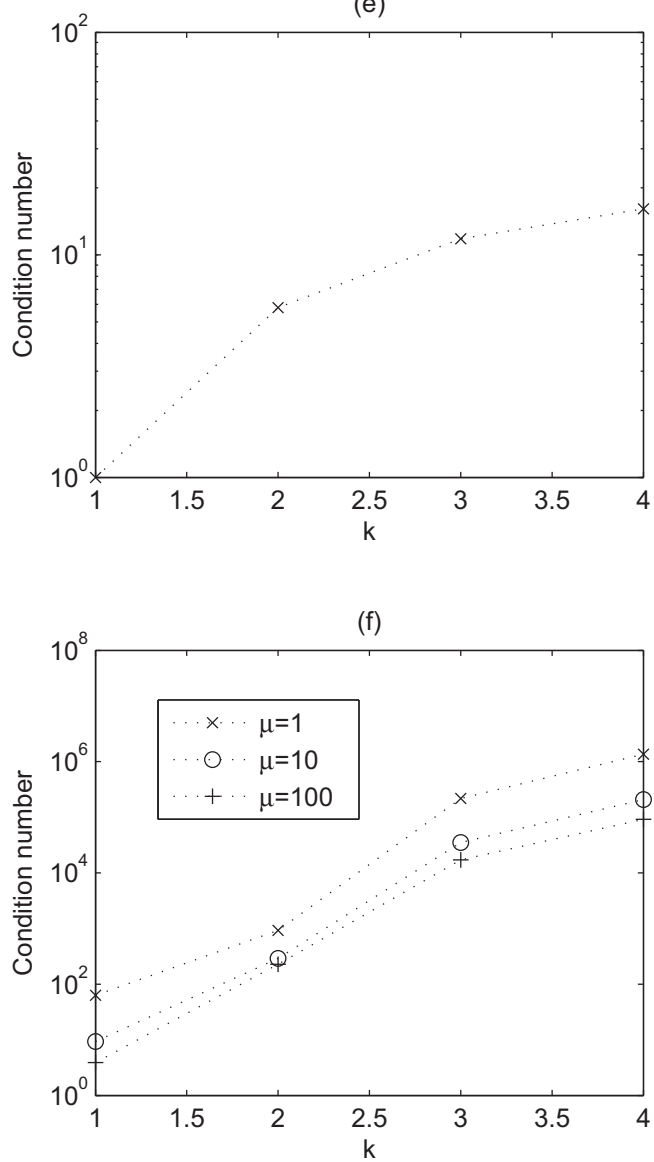

Figure 8: Condition number vs order for single element mass matrices, quasi-stiffness matrices and composite matrices for 1 -form bases ((a), (b), (c )) and 2-form bases $((d),(e),(f))$ on pyramid. 


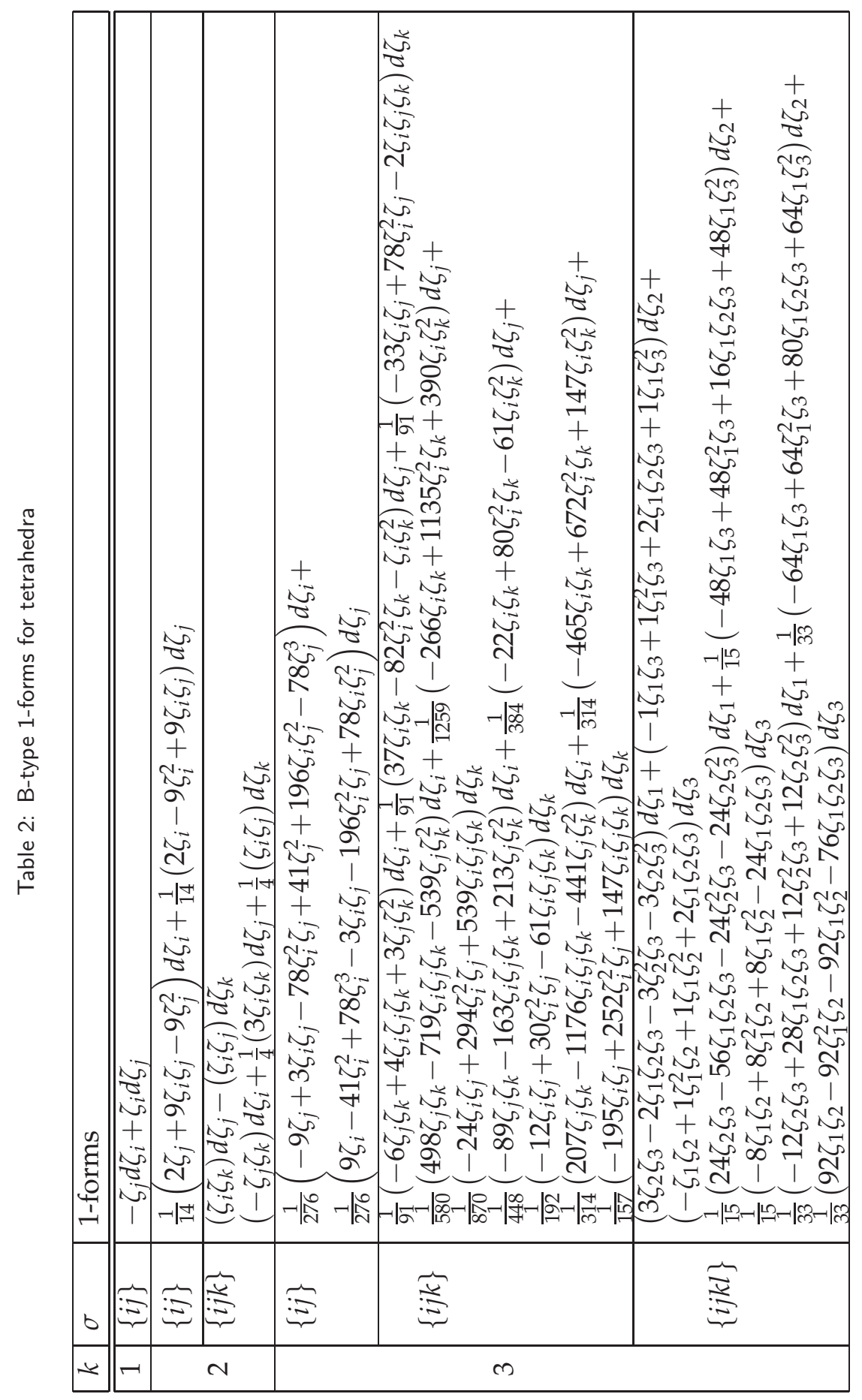




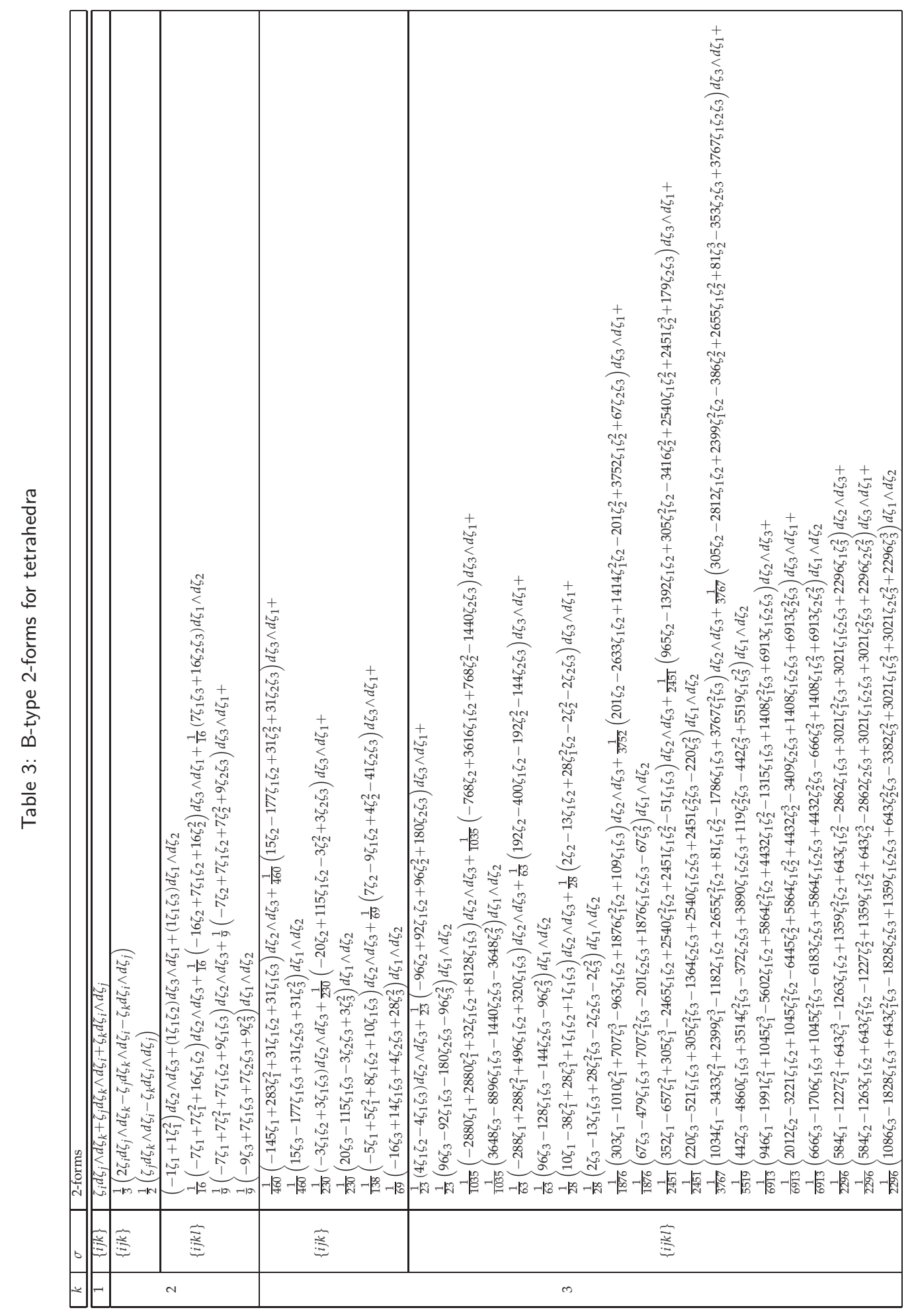


Table 4: 1-form and 2-form bases for tetrahedra (C-type)

\begin{tabular}{|c|c|c|}
\hline$k$ & $\sigma$ & 1-forms \\
\hline$\overline{11}$ & 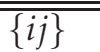 & $-\zeta_{j} d \zeta_{i}+\zeta_{i} d \zeta_{j}$ \\
\hline 2 & $\{i j\}$ & $\frac{1}{2}\left(-\zeta_{j} d \zeta_{i}-\zeta_{i} d \zeta_{j}\right)$ \\
\hline \multirow[b]{2}{*}{3} & $\{i j\}$ & $\frac{1}{12}\left(\zeta_{j}+3 \zeta_{i} \zeta_{j}-3 \zeta_{j}^{2}\right) d \zeta_{i}+\frac{1}{12}\left(-\zeta_{i}+3 \zeta_{i}^{2}-3 \zeta_{i} \zeta_{j}\right) d \zeta_{j}$ \\
\hline & $\{i j k\}$ & $\begin{array}{l}-\zeta_{j} \zeta_{k} d \zeta_{i}-\zeta_{i} \zeta_{k} d \zeta_{j}-\zeta_{i} \zeta_{j} d \zeta_{k} \\
\frac{1}{4}\left(\zeta_{j} \zeta_{k} d \zeta_{i}+5 \zeta_{i} \zeta_{k} d \zeta_{j}-3 \zeta_{i} \zeta_{j} d \zeta_{k}\right) \\
\frac{3}{31}\left(-11 \zeta_{j} \zeta_{k} d \zeta_{i}+7 \zeta_{i} \zeta_{k} d \zeta_{j}+2 \zeta_{i} \zeta_{j} d \zeta_{k}\right)\end{array}$ \\
\hline $\bar{k}$ & 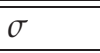 & 2-forms \\
\hline 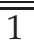 & 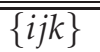 & 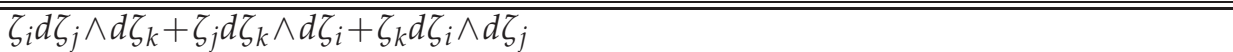 \\
\hline 2 & $\{i j k\}$ & $\begin{array}{l}\frac{1}{3}\left(2 \zeta_{i} d \zeta_{j} \wedge d \zeta_{k}-\zeta_{j} d \zeta_{k} \wedge d \zeta_{i}-\zeta_{k} d \zeta_{i} \wedge d \zeta_{j}\right) \\
\frac{1}{2}\left(\zeta_{j} d \zeta_{k} \wedge d \zeta_{i}-\zeta_{k} d \zeta_{i} \wedge d \zeta_{j}\right)\end{array}$ \\
\hline & $\{i j k\}$ & $\begin{array}{l}\frac{1}{5}\left(-\zeta_{i}+2 \zeta_{i}^{2}\right) d \zeta_{j} \wedge d \zeta_{k}+\frac{1}{10}\left(\zeta_{j}-5 \zeta_{i} \zeta_{j}\right) d \zeta_{k} \wedge d \zeta_{i}+\frac{1}{10}\left(\zeta_{k}-5 \zeta_{i} \zeta_{k}\right) d \zeta_{i} \wedge d \zeta_{j} \\
\frac{1}{10}\left(-\zeta_{j}+5 \zeta_{i} \zeta_{j}\right) d \zeta_{k} \wedge d \zeta_{i}+\frac{1}{10}\left(\zeta_{k}-5 \zeta_{i} \zeta_{k}\right) d \zeta_{i} \wedge d \zeta_{j} \\
\frac{1}{6}\left(\zeta_{j}-\zeta_{i} \zeta_{j}-4 \zeta_{j} \zeta_{k}\right) d \zeta_{k} \wedge d \zeta_{i}+\frac{1}{6}\left(-\zeta_{k}+\zeta_{i} \zeta_{k}+2 \zeta_{k}^{2}\right) d \zeta_{i} \wedge d \zeta_{j}\end{array}$ \\
\hline 3 & $\{i j k l\}$ & $\begin{array}{l}4\left(-\zeta_{2}+\zeta_{1} \zeta_{2}+\zeta_{2}^{2}+2 \zeta_{2} \zeta_{3}\right) d \zeta_{3} \wedge d \zeta_{1}+4\left(\zeta_{3}-\zeta_{1} \zeta_{3}-2 \zeta_{2} \zeta_{3}-\zeta_{3}^{2}\right) d \zeta_{1} \wedge d \zeta_{2} \\
\frac{8}{3}\left(-\zeta_{1}+\zeta_{1}^{2}+3 \zeta_{1} \zeta_{3}\right) d \zeta_{2} \wedge d \zeta_{3}+\frac{2}{3}\left(-\zeta_{2}+5 \zeta_{1} \zeta_{2}+\zeta_{2}^{2}-2 \zeta_{2} \zeta_{3}\right) d \zeta_{3} \wedge d \zeta_{1} \\
+\frac{2}{3}\left(5 \zeta_{3}-13 \zeta_{1} \zeta_{3}-2 \zeta_{2} \zeta_{3}-5 \zeta_{3}^{2}\right) d \zeta_{1} \wedge d \zeta_{2} \\
\frac{8}{11}\left(-6 \zeta_{1}+6 \zeta_{1}^{2}+11 \zeta_{1} \zeta_{2}+7 \zeta_{1} \zeta_{3}\right) d \zeta_{2} \wedge d \zeta_{3}+\frac{8}{11}\left(4 \zeta_{2}-9 \zeta_{1} \zeta_{2}-4 \zeta_{2}^{2}-3 \zeta_{2} \zeta_{3}\right) d \zeta_{3} \wedge d \zeta_{1} \\
+\frac{8}{11}\left(2 \zeta_{3}-3 \zeta_{1} \zeta_{3}-3 \zeta_{2} \zeta_{3}-2 \zeta_{3}^{2}\right) d \zeta_{1} \wedge d \zeta_{2} \\
\frac{1}{5}\left(-7 \zeta_{1}+7 \zeta_{1}^{2}+3 \zeta_{1} \zeta_{2}+3 \zeta_{1} \zeta_{3}\right) d \zeta_{2} \wedge d \zeta_{3}+\frac{1}{5}\left(1 \zeta_{2}+3 \zeta_{1} \zeta_{2}-1 \zeta_{2}^{2}-1 \zeta_{2} \zeta_{3}\right) d \zeta_{3} \wedge d \zeta_{1} \\
+\frac{1}{5}\left(1 \zeta_{3}+3 \zeta_{1} \zeta_{3}-1 \zeta_{2} \zeta_{3}-1 \zeta_{3}^{2}\right) d \zeta_{1} \wedge d \zeta_{2} \\
\frac{1}{65}\left(-27 \zeta_{1}+27 \zeta_{1}^{2}+56 \zeta_{1} \zeta_{2}+4 \zeta_{1} \zeta_{3}\right) d \zeta_{2} \wedge d \zeta_{3} \\
+\frac{4}{195}\left(-64 \zeta_{2}+42 \zeta_{1} \zeta_{2}+64 \zeta_{2}^{2}+25 \zeta_{2} \zeta_{3}\right) d \zeta_{3} \wedge d \zeta_{1} \\
+\frac{4}{195}\left(14 \zeta_{3}+3 \zeta_{1} \zeta_{3}+25 \zeta_{2} \zeta_{3}-14 \zeta_{3}^{2}\right) d \zeta_{1} \wedge d \zeta_{2} \\
\frac{2}{11}\left(-4 \zeta_{1}+4 \zeta_{1}^{2}+4 \zeta_{1} \zeta_{2}+5 \zeta_{1} \zeta_{3}\right) d \zeta_{2} \wedge d \zeta_{3}+\frac{2}{11}\left(-4 \zeta_{2}+4 \zeta_{1} \zeta_{2}+4 \zeta_{2}^{2}+5 \zeta_{2} \zeta_{3}\right) d \zeta_{3} \wedge d \zeta_{1} \\
\frac{2}{11}\left(-6 \zeta_{3}+5 \zeta_{1} \zeta_{3}+5 \zeta_{2} \zeta_{3}+6 \zeta_{3}^{2}\right) d \zeta_{1} \wedge d \zeta_{2}\end{array}$ \\
\hline
\end{tabular}


Table 5: 1-form and 2-form bases for hexahedra

\begin{tabular}{|c|c|c|}
\hline$k$ & $\sigma$ & 1-forms \\
\hline 1 & $\sigma_{1,7}$ & $\zeta_{1} \zeta_{2} d \zeta_{3}$ \\
\hline \multirow{3}{*}{2} & $\sigma_{1,7}$ & $\frac{1}{4} \zeta_{1} \zeta_{2}\left(5 \zeta_{2}+5 \zeta_{1}-8\right)\left(2 \zeta_{3}-1\right) d \zeta_{3}$ \\
\hline & $\sigma_{2,1}$ & $\begin{array}{l}\zeta_{2}\left(\zeta_{2}-1\right)\left(\zeta_{3}-1\right)\left(\zeta_{3}+1\right) d \zeta_{1} \\
\frac{1}{2} \zeta_{2}\left(\zeta_{2}-1\right)\left(\zeta_{3}-1\right)\left(\zeta_{2}-2 \zeta_{1}+1\right) d \zeta_{1} \\
\zeta_{1}\left(1-\zeta_{1}\right)\left(\zeta_{3}-1\right) d \zeta_{2} \\
\frac{1}{2} \zeta_{1}\left(1-\zeta_{1}\right)\left(2 \zeta_{1}-1\right)\left(\zeta_{3}-1\right) d \zeta_{2}\end{array}$ \\
\hline & $\sigma_{3}$ & $\begin{array}{l}-\zeta_{1} \zeta_{2} \zeta_{3}\left(\zeta_{2}-1\right)\left(\zeta_{3}-1\right) d \zeta_{1} \\
\zeta_{1} \zeta_{3}\left(\zeta_{1}-1\right)\left(\zeta_{3}-1\right) d \zeta_{2} \\
\frac{1}{2} \zeta_{2} \zeta_{3}\left(3 \zeta_{1}-2\right)\left(\zeta_{2}-1\right)\left(\zeta_{3}-1\right) d \zeta_{1} \\
\frac{1}{2} \zeta_{1} \zeta_{3}\left(\zeta_{1}-1\right)\left(2 \zeta_{2}-1\right)\left(\zeta_{3}-1\right) d \zeta_{2} \\
\zeta_{1} \zeta_{2}\left(\zeta_{1}-1\right)\left(\zeta_{2}-1\right) d \zeta_{3} \\
\frac{1}{2} \zeta_{1} \zeta_{2}\left(\zeta_{1}-1\right)\left(\zeta_{2}-1\right)\left(2 \zeta_{3}-1\right) d \zeta_{3}\end{array}$ \\
\hline $\bar{k}$ & $\sigma$ & 2-forms \\
\hline 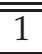 & $\sigma_{2,1}$ & $\left(1-\zeta_{3}\right) d \zeta_{1} \wedge d \zeta_{2}$ \\
\hline \multirow[b]{2}{*}{2} & $\sigma_{2,1}$ & $\begin{array}{l}\frac{1}{2}\left(2 \zeta_{2}-1\right)\left(1-\zeta_{3}\right) d \zeta_{1} \wedge d \zeta_{2} \\
\frac{1}{2}\left(2 \zeta_{1}-1\right)\left(1-\zeta_{3}\right) d \zeta_{1} \wedge d \zeta_{2} \\
\frac{1}{4}\left(2 \zeta_{1}-1\right)\left(2 \zeta_{2}-1\right)\left(1-\zeta_{3}\right) d \zeta_{1} \wedge d \zeta_{2}\end{array}$ \\
\hline & $\sigma_{3}$ & $\begin{array}{l}\zeta_{1} \zeta_{2}\left(\zeta_{2}-1\right) d \zeta_{3} \wedge d \zeta_{1} \\
\frac{1}{2} \zeta_{1}\left(\zeta_{2}-1\right) \zeta_{2}\left(2 \zeta_{3}-1\right) d \zeta_{3} \wedge d \zeta_{1} \\
\zeta_{1} \zeta_{3}\left(\zeta_{3}-1\right) d \zeta_{2} \wedge d \zeta_{3} \\
\frac{1}{2} \zeta_{1}\left(\zeta_{1}-1\right)\left(3 \zeta_{3}-2\right) d \zeta_{2} \wedge d \zeta_{3} \\
\frac{1}{2} \zeta_{2} \zeta_{3}\left(3 \zeta_{1}-2\right)\left(1-\zeta_{2}\right) d \zeta_{3} \wedge d \zeta_{1} \\
\zeta_{2} \zeta_{3}\left(\zeta_{3}-1\right) d \zeta_{1} \wedge d \zeta_{2} \\
\frac{1}{2} \zeta_{3}\left(\zeta_{3}-1\right)\left(3 \zeta_{2}-2\right) d \zeta_{1} \wedge d \zeta_{2} \\
\frac{1}{2} \zeta_{1}\left(\zeta_{1}-1\right)\left(2 \zeta_{2}-1\right) d \zeta_{2} \wedge d \zeta_{3} \\
\frac{1}{2} \zeta_{3}\left(\zeta_{3}-1\right)\left(2 \zeta_{1}-1\right) d \zeta_{1} \wedge d \zeta_{2} \\
\frac{1}{4}\left(3 \zeta_{1}-2\right)\left(1-\zeta_{2}\right)\left(3 \zeta_{3}-2\right) d \zeta_{3} \wedge d \zeta_{1} \\
\frac{1}{4} \zeta_{1}\left(\zeta_{1}-1\right)\left(2 \zeta_{2}-1\right)\left(2 \zeta_{3}-1\right) d \zeta_{2} \wedge d \zeta_{3} \\
\frac{1}{4} \zeta_{3}\left(2 \zeta_{1}-1\right)\left(2 \zeta_{2}-1\right)\left(\zeta_{3}-1\right) d \zeta_{1} \wedge d \zeta_{2}\end{array}$ \\
\hline
\end{tabular}




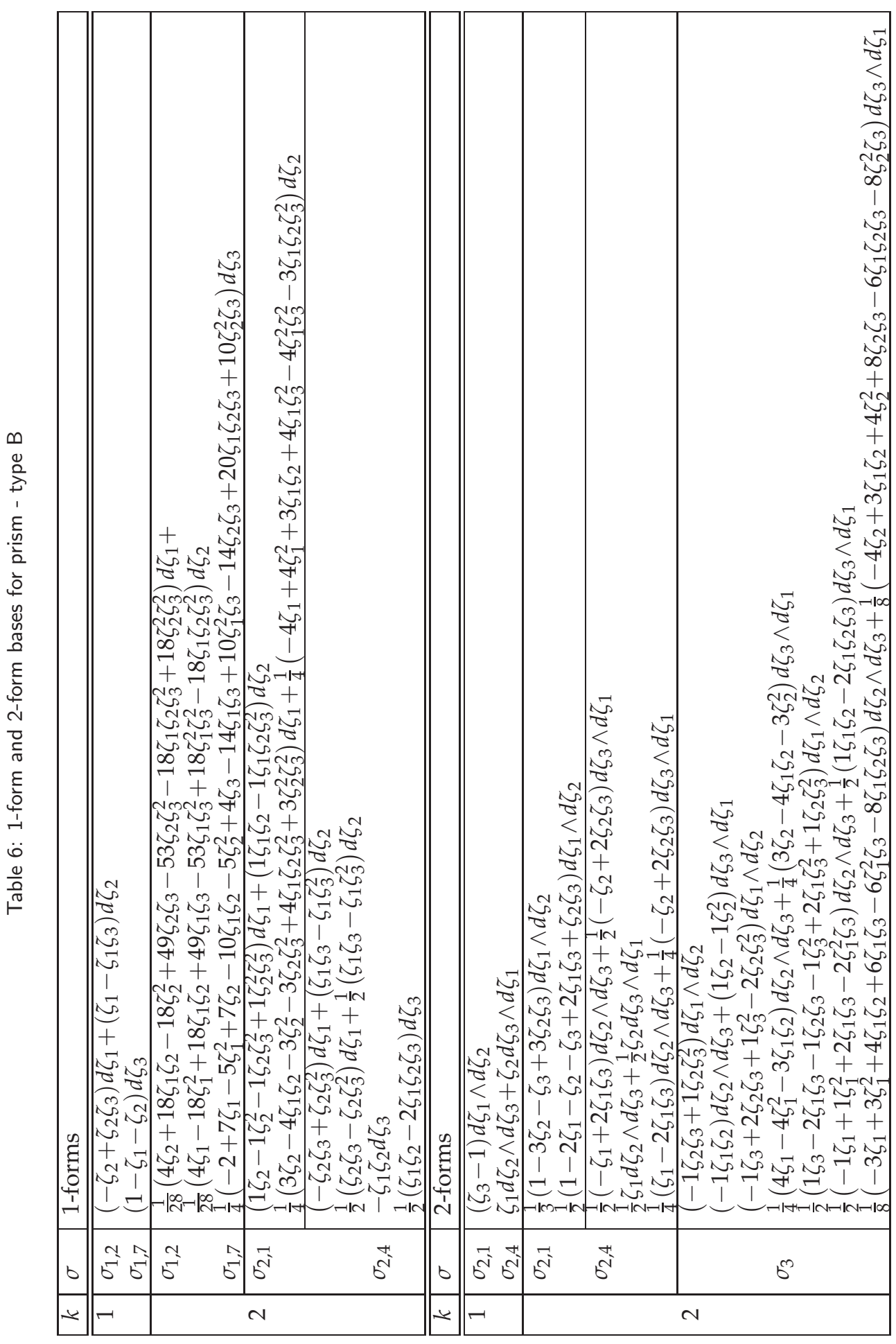


Table 7: 1-form and 2-form bases for prism - type C

\begin{tabular}{|c|c|c|}
\hline$k$ & $\sigma$ & 1-forms \\
\hline $\bar{~} 1$ & $\begin{array}{l}\sigma_{1,2} \\
\sigma_{1,7}\end{array}$ & $\begin{array}{l}\left(-\zeta_{2}+\zeta_{2} \zeta_{3}\right) d \zeta_{1}+\left(\zeta_{1}-\zeta_{1} \zeta_{3}\right) d \zeta_{2} \\
\left(1-\zeta_{1}-\zeta_{2}\right) d \zeta_{3}\end{array}$ \\
\hline & $\begin{array}{l}\sigma_{1,2} \\
\sigma_{1,7}\end{array}$ & $\begin{array}{l}\frac{1}{4}\left(-2 \zeta_{2}+7 \zeta_{2} \zeta_{3}-5 \zeta_{2} \zeta_{3}^{2}\right) d \zeta_{1}+\frac{1}{4}\left(-2 \zeta_{1}+7 \zeta_{1} \zeta_{3}-5 \zeta_{1} \zeta_{3}^{2}\right) d \zeta_{2} \\
\frac{1}{4}\left(\zeta_{1}+\zeta_{2}-1\right)\left(5 \zeta_{1}+5 \zeta_{2}-2\right)\left(2 \zeta_{3}-1\right) d \zeta_{3}\end{array}$ \\
\hline 2 & $\sigma_{2,4}$ & $\begin{array}{l}\left(-\zeta_{2} \zeta_{3}+\zeta_{2} \zeta_{3}^{2}\right) d \zeta_{1}+\left(\zeta_{1} \zeta_{3}-\zeta_{1} \zeta_{3}^{2}\right) d \zeta_{2} \\
\frac{1}{2}\left(\zeta_{2} \zeta_{3}-\zeta_{2} \zeta_{3}^{2}\right) d \zeta_{1}+\frac{1}{2}\left(\zeta_{1} \zeta_{3}-\zeta_{1} \zeta_{3}^{2}\right) d \zeta_{2} \\
-\zeta_{1} \zeta_{2} d \zeta_{3} \\
\frac{1}{2}\left(\zeta_{1} \zeta_{2}-2 \zeta_{1} \zeta_{2} \zeta_{3}\right) d \zeta_{3}\end{array}$ \\
\hline $\bar{k}$ & $\bar{\sigma}$ & 2-forms \\
\hline$\overline{11}$ & $\begin{array}{l}\sigma_{2,1} \\
\sigma_{2,4}\end{array}$ & $\begin{array}{l}\left(\zeta_{3}-1\right) d \zeta_{1} \wedge d \zeta_{2} \\
\zeta_{1} d \zeta_{2} \wedge d \zeta_{3}+\zeta_{2} d \zeta_{3} \wedge d \zeta_{1}\end{array}$ \\
\hline \multirow[t]{2}{*}{2} & $\sigma_{2,1}$ & $\begin{array}{l}\frac{1}{3}\left(1-3 \zeta_{2}-\zeta_{3}+3 \zeta_{2} \zeta_{3}\right) d \zeta_{1} \wedge d \zeta_{2} \\
\frac{1}{2}\left(1-2 \zeta_{1}-\zeta_{2}-\zeta_{3}+2 \zeta_{1} \zeta_{3}+\zeta_{2} \zeta_{3}\right) d \zeta_{1} \wedge d \zeta_{2} \\
\frac{1}{2}\left(-\zeta_{1}+2 \zeta_{1} \zeta_{3}\right) d \zeta_{2} \wedge d \zeta_{3}+\frac{1}{2}\left(-\zeta_{2}+2 \zeta_{2} \zeta_{3}\right) d \zeta_{3} \wedge d \zeta_{1} \\
\frac{1}{2} \zeta_{1} d \zeta_{2} \wedge d \zeta_{3}+\frac{1}{2} \zeta_{2} d \zeta_{3} \wedge d \zeta_{1} \\
\frac{1}{4}\left(\zeta_{1}-2 \zeta_{1} \zeta_{3}\right) d \zeta_{2} \wedge d \zeta_{3}+\frac{1}{4}\left(-\zeta_{2}+2 \zeta_{2} \zeta_{3}\right) d \zeta_{3} \wedge d \zeta_{1}\end{array}$ \\
\hline & $\sigma_{3}$ & $\begin{array}{l}\left(-\zeta_{3}+\zeta_{3}^{2}\right) d \zeta_{1} \wedge d \zeta_{2} \\
\frac{1}{3}\left(\zeta_{3}-3 \zeta_{1} \zeta_{3}-\zeta_{3}^{2}+3 \zeta_{1} \zeta_{3}^{2}\right) d \zeta_{1} \wedge d \zeta_{2} \\
\frac{1}{2}\left(\zeta_{3}-\zeta_{1} \zeta_{3}-2 \zeta_{2} \zeta_{3}-\zeta_{3}^{2}+\zeta_{1} \zeta_{3}^{2}+2 \zeta_{2} \zeta_{3}^{2}\right) d \zeta_{1} \wedge d \zeta_{2}\end{array}$ \\
\hline
\end{tabular}




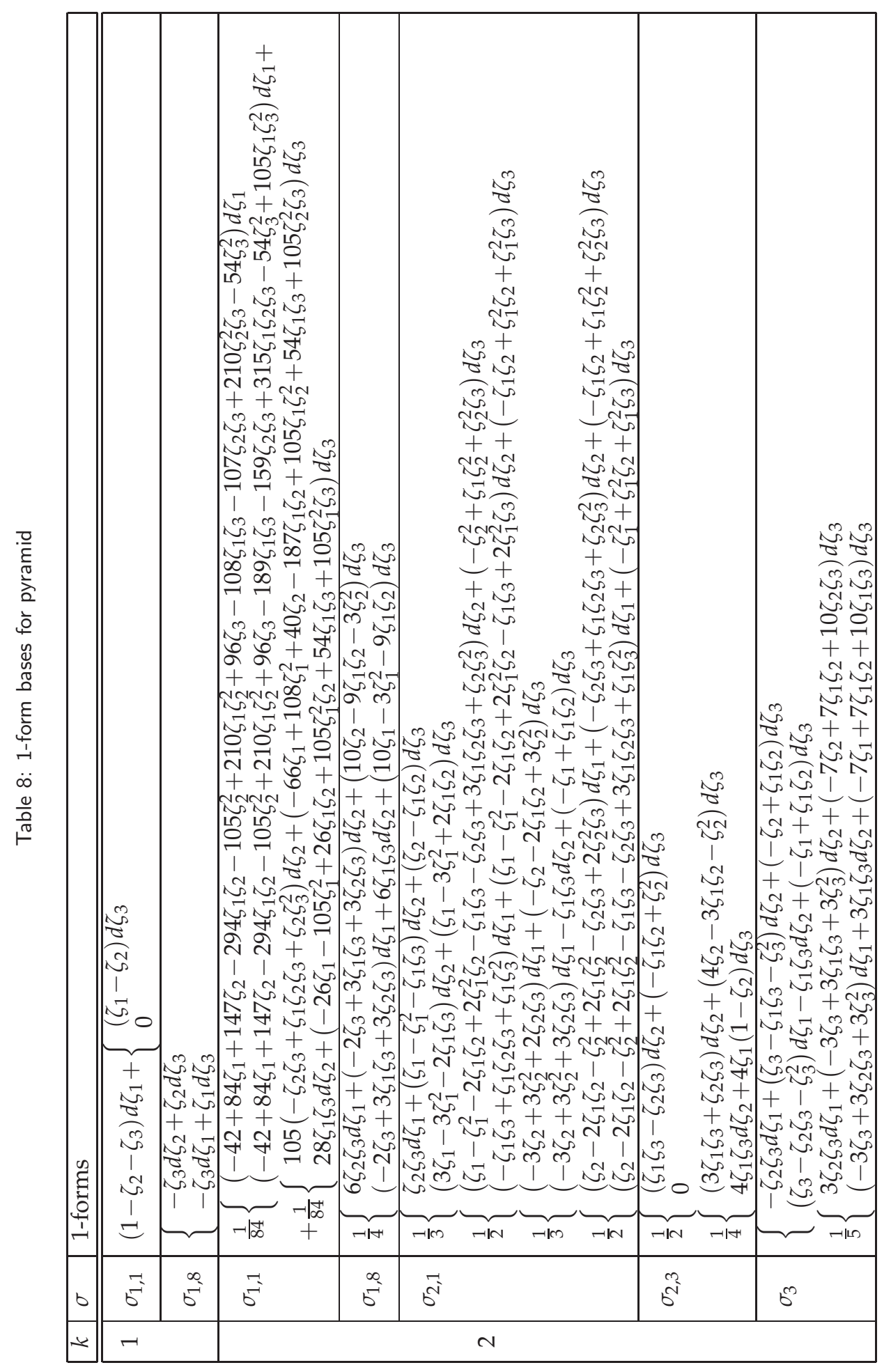




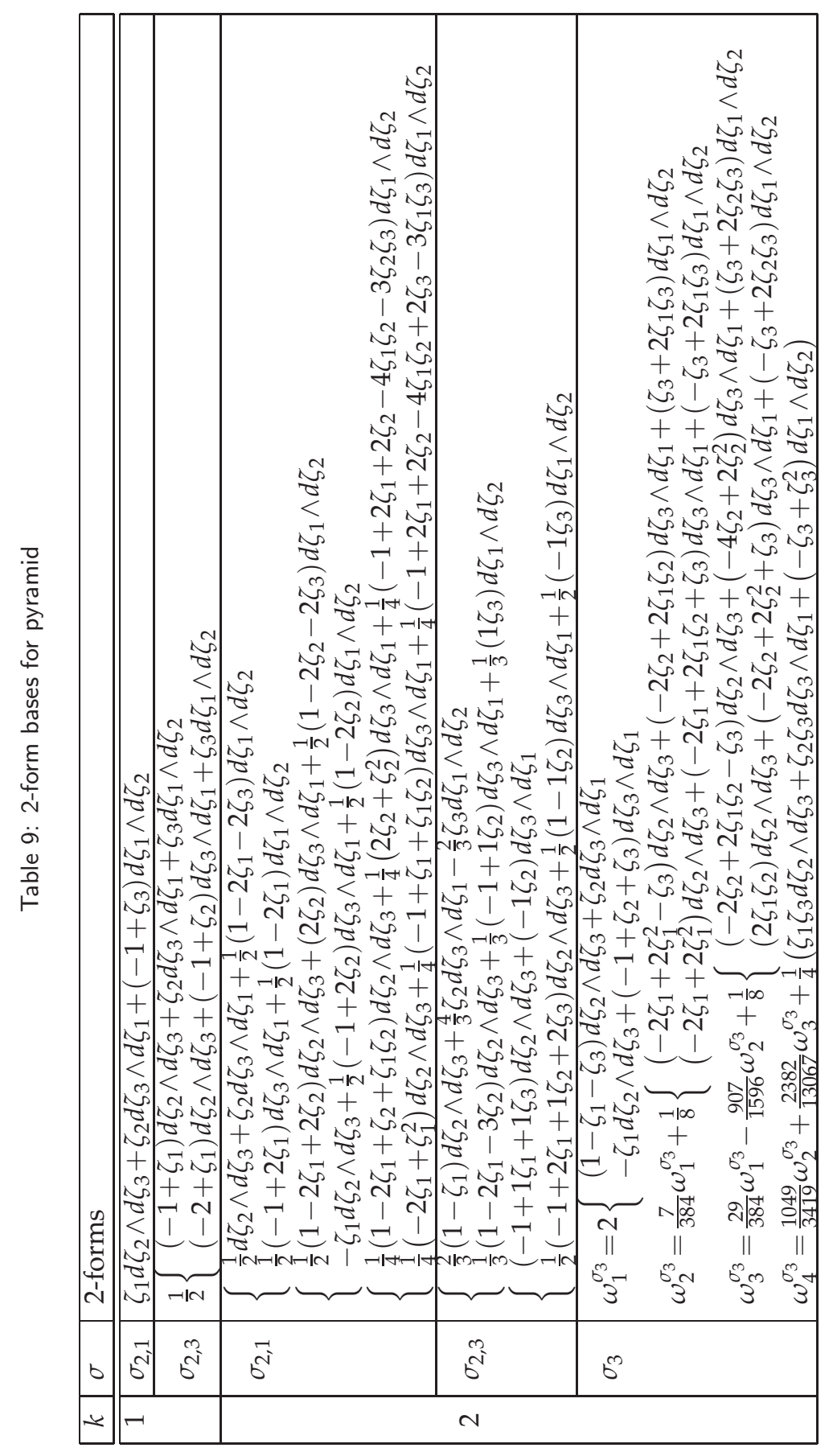

